Bull. Soc. math. France

129 (4), 2001, p. 593-623

\title{
TRIPLETS SPECTRAUX POUR LES VARIÉTÉS À SINGULARITÉ CONIQUE ISOLÉE
}

\author{
PAR JeAN-Marie LESCURE
}

\begin{abstract}
RÉSumÉ. - Sur une pseudo-variété de dimension paire à une singularité conique isolée, des triplets spectraux sont construits à partir d'une classe d'opérateurs différentiels elliptiques de type Fuchs, contenant les opérateurs de Dirac à coefficients dans des fibrés plats dans la direction radiale. Ces derniers engendrent, sous une hypothèse raisonnable, le groupe de $K$-homologie pair tensorisé par $\mathbb{C}$ de la pseudo-variété et leur caractère de Chern est calculé.

ABSTRACT (Spectral triples for pseudomanifolds with isolated singularity)

We use elliptic operators of Fuchs type on an even dimensional pseudomanifold with an isolated singularity to construct spectral triples. This class of operators includes Dirac operators with coefficients in flat bundles in the radial direction and, under some hypothesis, these operators generate the even $K$-homology group tensorised by $\mathbb{C}$ of the pseudomanifold. Moreover, their Chern character is computed.
\end{abstract}

\section{Table des matières}

1. Introduction. ....................................... 594

2. Triplets spectraux................................ 596

3. Pseudo-variétés à une singularité conique... . . . . . . . . . . . . . . . 598

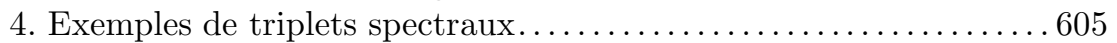

5. Calcul du caractère de Chern.........................6 616

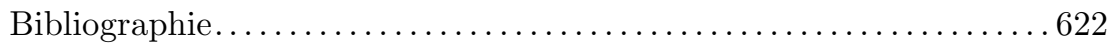

Texte reçu le 7 avril 2000, révisé le 25 janvier 2001 et le 13 juin 2001

Jean-Marie Lescure, Département de Mathématiques, Université Blaise Pascal, Complexe universitaire des Cézeaux, 63177 Aubière CEDEX

E-mail : lescure@math.univ-bpclermont.fr

Classification mathématique par sujets (2000). — 19D55, 58J42.

Mots clefs. - Formule locale de l'indice, variétés singulières. 


\section{Introduction}

Le théorème de l'indice de M.F. Atiyah et I.M. Singer figure parmi les résultats fondamentaux de ces dernières décennies. Il rencontre de nombreux champs de recherches, et a fait l'objet de nombreuses généralisations.

Un des aspects fondamentaux de la géométrie non commutative est le calcul du caractère de Chern des $K$-cycles, ce qui correspond à un problème de l'indice généralisé. En effet, un $K$-cycle $(D, \mathcal{H})$ sur une $(\mathbb{C}$-)algèbre $\mathcal{A}$ induit une application dite indice analytique :

$$
\operatorname{Ind}_{D}: K_{j}(\mathcal{A}) \longrightarrow \mathbb{Z},
$$

où $j$ dépend de la parité du $K$-cycle. Pour calculer cette application, A. Connes a introduit la cohomologie cyclique $H C^{*}(\mathcal{A})$, réceptacle naturel du caractère de Chern $\mathrm{Ch}^{j}(D)$ qu'il définit de sorte que

$$
\operatorname{Ind}_{D}([e])=\left\langle\mathrm{Ch}^{j}(D) ;[e]\right\rangle,
$$

où $\langle$,$\rangle est un pairing naturel entre la cohomologie cyclique et la K$-théorie. Le terme de droite est une formule cohomologique pour l'indice analytique et est un substitut de l'indice topologique de M. Atiyah et I. Singer. Une première formule explicite du caractère de Chern des $K$-cycles apparaît dans [11].

En 1995, A. Connes et H. Moscovici établissent dans [14] une nouvelle formule pour le caractère de Chern de certains $K$-cycles, qu'ils appellent des triplets spectraux. Leur travail comporte deux parties. Dans la première, ils définissent les triplets spectraux comme étant des $K$-cycles $(\mathcal{D}, \mathcal{H})$ sur l'algèbre $\mathcal{A}$ tels que les fonctions $z \mapsto \operatorname{Tr}\left(b|\mathcal{D}|^{z}\right)$ aient un bon prolongement méromorphe à $\mathbb{C}$, où $b$ est dans une algèbre $\mathcal{B}$ contenant $\mathcal{A}$ et stable par certains commutateurs. Cette notion est beaucoup plus fine et restrictive que celle de $K$-cycle [11], [12]. En contrepartie, ils obtiennent une formule « locale » du caractère de Chern sous la forme d'un cocycle du $(b, B)$-bicomplexe de $\mathcal{A}$. Rappelons que la cohomologie de ce bicomplexe est la cohomologie cyclique (périodique) de $\mathcal{A}$. Contrairement aux formules antérieures, définies comme des traces de produit d'opérateurs, donc d'intégrales de quantités non locales, leur formule est basée sur des résidus de fonctions méromorphes qui généralisent le résidu de Wodzicki et qui sont des intégrales de quantités locales.

A. Connes et H. Moscovici traitent dans leur article [14] le cas des feuilletages et mentionnent dans l'introduction, parmi les exemples possibles, le cas des variétés à singularités coniques.

C'est cet exemple que nous traitons dans ce travail. Avant d'expliquer plus en détail le contenu, il est utile de comparer l'approche de A. Connes et H. Moscovici et celle de J. Cheeger, J. Brüning et R. Seeley.

Le travail de A. Connes et $\mathrm{H}$. Moscovici fournit un cadre et une réponse généraux, pour le problème de l'indice en géométrie non commutative. Cette réponse donne plus que l'indice du $K$-cycle. En effet, le caractère de Chern

TOME $129-2001-\mathrm{N}^{\mathrm{O}} 4$ 
donne l'indice de tous les $K$-cycles résultant du pairing entre la $K$-homologie et la $K$-théorie; une bonne idée de ce pairing est donnée (dans la cas d'une variété) par l'indice des opérateurs elliptiques à coefficients dans des fibrés, les opérateurs elliptiques étant les cycles de la $K$-homologie et les fibrés les cycles de la $K$-théorie. On peut aussi noter que le caractère de Chern est une classe de cohomologie cyclique dont la composante de degré 0 correspond à l'indice du $K$-cycle.

J. Cheeger, J. Brüning et R. Seeley, M. Lesch, ont établi des formules de l'indice pour une large classe d'opérateurs elliptiques sur les variétés singulières. Ils étudient les propriétés de Fredholm de certaines extensions auto-adjointes, construisent les noyaux de la chaleur et les résolvantes correspondants, puis obtiennent les développements asymptotiques de leur trace. Ces développements ne résultent pas de l'intégration des développements ponctuels comme dans la cas des variétés compactes sans bord : les coefficients du développement asymptotique ponctuel du noyau de la chaleur ne sont pas tous intégrables. Aussi, ils établissent et utilisent des lemmes de « développements asymptotiques singuliers » (S.A.L. [5], [22]) comportant une régularisation des intégrales divergentes.

Prouver que des $K$-cycles sont des triplets spectraux nécessite dans chaque cas des méthodes spécifiques et pour le cas des variétés singulières, les travaux de [9], [6], [4] sont pertinents, ce qui est d'ailleurs noté dans l'introduction de $[14]$.

Le présent travail met donc en évidence la complémentarité entre l'approche de A. Connes-H. Moscovici et celle de J. Cheeger J. Brüning, R. Seeley et M. Lesch : partant du travail de ces derniers, on peut construire des triplets spectraux, puis calculer leur caractère de Chern à l'aide du travail de A. Connes et H. Moscovici. Cela donne par exemple des informations sur la $K$-homologie : on montre que la $K$-homologie sans torsion (des variétés à singularités coniques) est obtenue à l'aide des opérateurs de Dirac à coefficients.

Nous terminons cette introduction par un rapide résumé de l'article. Nous construisons des triplets spectraux à partir d'extensions auto-adjointes d'opérateurs différentiels de Fuchs elliptiques définis sur la partie lisse $X$ d'une pseudo-variété compacte à une singularité conique $X^{c}$. Ils forment une classe naturelle et ont été largement étudiés [8], [9], [6], [22], [4], [27]. En particulier les opérateurs géométriques associés à un métrique conique sont de ce type.

Pour prouver l'existence des prolongements méromorphes requis, nous séparons ce qui vient de la partie lisse et ce qui vient de la singularité. Cette dernière contribution est traitée à l'aide de résultats techniques découlant d'un plongement de Sobolev à poids. C'est une approche directe et en grande partie auto-contenue, mais nous aurions pu aussi utiliser [4], ou encore [17].

Parmi les triplets spectraux obtenus figurent ceux provenant des opérateurs de Dirac à coefficients dans des fibrés munis d'une structure produit sur le 
bout cylindrique de la variété $X$. Nous calculons explicitement leur caractère de Chern à partir de la formule de Connes-Moscovici. Le travail de [6], [22] donne la composante de degré 0 du cocycle et pour les composantes en degré positif, nous utilisons le calcul de E. Getzler [16], [13], [2] après nous être ramené au cas d'une variété compacte à l'aide d'une « excision » des contributions singulières. Nous montrons ensuite, c'est une conséquence des formules établies, que nous avons ainsi suffisamment de $K$-cycles pour engendrer la partie sans torsion de la $K$-homologie de $X^{c}$.

L'algèbre $\mathcal{A}$ choisie est l'algèbre des fonctions $C^{\infty}$ sur la partie lisse $X$ de $X^{c}$ et constantes près de la singularité. Ce choix a plusieurs avantages. Tout d'abord, l'algèbre $\mathcal{A}$ est suffisamment grande : elle est dense et stable par calcul fonctionnel holomorphe dans $C\left(X^{c}\right)$ (les fonctions continues sur $X^{c}$ ), donc a même $K$-théorie que $C\left(X^{c}\right)$. De même, nous établissons que sa cohomologie cyclique est isomorphe à l'homologie usuelle de $X^{c}$. Ensuite, elle est suffisamment petite : dès qu'apparaît une dérivation ou un commutateur dans les calculs, on est ramené à un travail sur une partie compacte de $X$, où les choses sont plus classiques. En outre, la structure particulière de cette algèbre autorise la séparation en contribution des parties régulières et singulières et « l'excision » de la singularité dont nous avons parlé plus haut. Enfin, elle admet des généralisations naturelles à des stratifications plus complexes (les constantes devenant des fonctions $C^{\infty}$ sur les strates...), pour lesquelles nous espérons tirer partie des avantages décrits ci-dessus dans un travail à venir. Dans un souci de brièveté, certaines preuves techniques ont été omises. Cela ne devrait pas entraver la compréhension de l'article, toutefois, le lecteur peut consulter ces preuves dans [23].

Nous terminons en citant les articles de H. Moscovici et F. Wu [25] et de S. Chan [7] où des formules du caractère de Chern de l'opérateur de la signature d'une pseudo-variété sont données. Leurs méthodes et les opérateurs considérés sont différents et ils ne font pas usage de la formule locale de l'indice.

\section{Triplets spectraux}

Soient $\mathcal{A}$ une $\mathbb{C}$-algèbre involutive, $\mathcal{H}$ un espace de Hilbert, $\rho$ une représentation de $\mathcal{A}$ sur $\mathcal{H}$ et $\mathcal{D}$ un opérateur auto-adjoint non borné sur $\mathcal{H}$. On dit que $(\mathcal{D}, \mathcal{H}, \rho)$ est un module de Fredholm non borné ou un $K$-cycle sur $\mathcal{A}$ si :

$$
\begin{aligned}
\forall a \in \mathcal{A}, \quad[\mathcal{D}, \rho(a)] & \in \mathcal{B}(\mathcal{H}), \\
\left(1+\mathcal{D}^{2}\right)^{-1} & \in \mathcal{K}(\mathcal{H}) .
\end{aligned}
$$

Nous avons noté ci-dessus $\mathcal{B}(\mathcal{H})$ la $C^{*}$-algèbre des opérateurs bornés sur $\mathcal{H}$ et $\mathcal{K}(\mathcal{H})$ celle des opérateurs compacts. Le $K$-cycle est $p$-sommable si $\left(1+\mathcal{D}^{2}\right)^{-1} \in \mathcal{L}_{p}(\mathcal{H})$, où $\mathcal{L}_{p}(\mathcal{H})$ est le $p$-ième idéal de Schatten. Le module est pair si $\mathcal{H}$ est $\mathbb{Z}_{2}$ gradué et $\mathcal{D}$ est de degré 1 tandis que $\rho$ est de degré 0 ( $\mathcal{A}$ ayant

TOME $129-2001-\mathrm{N}^{\mathrm{O}} 4$ 
par défaut la $\mathbb{Z}_{2}$-graduation triviale). L'application $\rho$ sera généralement omise dans les notations. Le lecteur intéressé par la géométrie non commutative pourra consulter, entre autres, les références suivantes : [12], [29], [19], [1].

La sommabilité d'un $K$-cycle correspond à sa dimension en tant que cycle de la $K$-homologie. Les notions de spectre des dimensions et de triplet spectral dues à A. Connes et H. Moscovici [14] et rappelées ci-dessous, précisent les composantes du $K$-cycle en deçà de cette dimension maximale.

Soit $\mathcal{D}$ un opérateur non borné auto-adjoint sur $\mathcal{H}$. Nous noterons $\delta_{\mathcal{D}}$ (et souvent simplement $\delta$ ), l'opérateur non borné sur $\mathcal{B}(\mathcal{H})$ suivant :

$$
\delta_{\mathcal{D}}(T):=[|\mathcal{D}|, T] .
$$

DÉfinition 2.1 (voir [14]). — Un triplet spectral est un $K$-cycle $(\mathcal{H}, \mathcal{D}, \mathcal{A})$ $p$-sommable sur $\mathcal{A}$ tel que

$$
\mathcal{A} \subset \bigcap_{n \in \mathbb{N}} \operatorname{Dom} \delta_{\mathcal{D}}^{n}
$$

et tel qu'il existe une partie discrète $S d \subset \mathbb{C}$ telle que pour tout élément de l'algèbre $\mathcal{B}$ engendrée par $\left\{\delta^{n}(a) ; a \in \mathcal{A}, n \in \mathbb{N}\right\}$, la fonction suivante

$$
h_{b}: z \longmapsto \operatorname{tr}\left(b|D|^{-z}\right),
$$

admet un prolongement holomorphe à $\mathbb{C} \backslash S d$ avec pôles de multiplicité finie. $S d$ est appelé le spectre des dimensions. La borne supérieure des multiplicités des pôles est appelée multiplicité du spectre des dimensions.

En fait, on peut vérifier que la condition $\left(C_{1}\right)$ est équivalente à

$$
\forall a \in \mathcal{A}, \forall p, q \in \mathbb{N}, \quad|\mathcal{D}|^{-p} \nabla^{p+q}(a)|\mathcal{D}|^{-q} \in \mathcal{B}(\mathcal{H}) .
$$

où on a posé $\nabla(a)=\left[\mathcal{D}^{2}, a\right]$. A. Connes et H. Moscovici ont établi, à partir de l'expression donnée dans [18], une formule du caractère de Chern des triplets spectraux. On la rappelle dans le cas pair :

ThÉORÈme 2.2 (voir [14]). — Soit $(\mathcal{H}, \mathcal{D}, \mathcal{A})$ un triplet spectral pair. Son caractère de Chern est représenté par le cocycle $\left(\varphi_{n}\right)_{n \in 2 \mathbb{N}} d u(b, B)$-bicomplexe de l'algèbre $\mathcal{A}$ suivant :

- Pour $n>0$,

(3) $\varphi_{n}\left(a_{0}, \ldots, a_{n}\right)$

$$
=\sum_{\substack{k=\left(k_{1}, \ldots, k_{n}\right) \in \mathbb{N}^{n} \\ q \in \mathbb{N}}} \sigma_{k, q, n} \tau_{q}\left(\gamma a_{0}\left(d a_{1}\right)^{\left(k_{1}\right)} \cdots\left(d a_{n}\right)^{\left(k_{n}\right)}|\mathcal{D}|^{-(2|k|+n)}\right),
$$

où $\gamma$ est l'opérateur de graduation, da $=[\mathcal{D}, a], \nabla(a)=\left[\mathcal{D}^{2}, a\right], \tau_{q}(P)$ est le résidu en 0 de la fonction $z^{q} \operatorname{Tr}\left(P|\mathcal{D}|^{-2 z}\right)$ et $|k|=\sum_{i=1}^{n} k_{i}$. Les coefficients $\sigma_{k, q, n}$ sont des rationnels et seul un nombre fini d'entre eux sont non nuls.

BULletin DE LA SOCiÉtÉ MATHÉmATiQUe DE FRANCE 
- Pour $n=0$,

$$
\varphi_{0}(a)=\tau_{-1}(\gamma a)+\operatorname{Tr}(\gamma a H),
$$

où $H$ est le projecteur orthogonal sur $\operatorname{ker} \mathcal{D}$.

\section{Pseudo-variétés à une singularité conique}

3.1. Notations. - Soit $M$ une variété $C^{\infty}$ compacte de dimension paire $m=n+1$ à bord connexe $N$. Formons la pseudo-variété :

$$
X^{c}=(([0,1] \times N) /(\{0\} \times N)) \cup M .
$$

Notons $c$ le sommet du cône et $X$ la variété lisse $X^{c} \backslash\{c\}$ qui sera munie d'une métrique $g$ égale à $d r^{2}+r^{2} g_{N}$ sur la partie conique ]0,1] $\times N$, où $g_{N}$ est la métrique induite par $g$ sur $\partial M=N$.

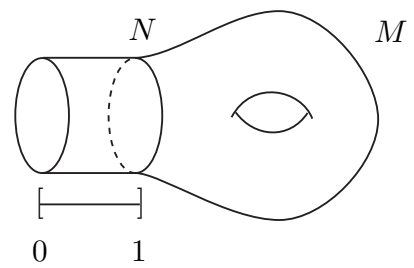

La variété $\bar{X}$

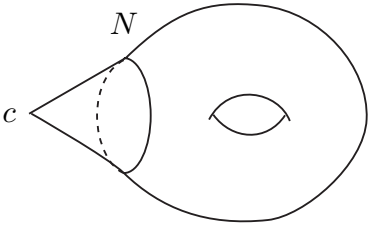

La variété conique $X^{c}$

Figure 1

Les recouvrements ouverts de $X$ seront obtenus en complétant un recouvrement ouvert fini $\left(O_{i}\right)_{i}$ de $M$ (les $O_{i}$ étant relativement compacts dans $X$ ) par des secteurs $] 0,1] \times U_{j}$ où $\left(U_{j}\right)_{j}$ est un recouvrement ouvert fini de $N$. Étant données des partitions de l'unité $\left(\theta_{j}\right)_{j}$ de $N$ subordonnée à $\left(U_{j}\right)_{j}$ et $\left(\psi_{i}\right)_{i}$ de $M$ subordonnée à $\left(O_{i}\right)_{i}$, étant donnée une fonction $\varphi \in C_{c}^{\infty}(X)$ égale à 1 sur $\bigcup_{i} O_{i}$, on obtient une partition de l'unité sur $X$ :

$$
1=(1-\varphi)+\varphi=(1-\varphi) \cdot\left(1 \otimes \sum_{j} \theta_{j}\right)+\varphi \sum_{i} \psi_{i} .
$$

3.2. Fibrés. - Nous considérerons des restrictions à $X$ de fibrés vectoriels $C^{\infty}$ complexes $E$ sur $\bar{X}=X \cup\{0\} \times N$. Si la restriction à $N$ d'un objet $W$ sur $X$ a un sens, nous la noterons $\widetilde{W}$. Nous noterons $C^{\infty}(E)$ l'espace des sections $C^{\infty} \operatorname{de} \mathrm{E}$ et $C_{c}^{\infty}(E)$ celui des sections à support compact. Sur la partie conique, les sections de $E$ sont canoniquement identifiées aux éléments de $\left.\left.C^{\infty}(] 0,1\right], C^{\infty}(\widetilde{E})\right)$. 
Soient $h=|\cdot|$ une métrique sur $E$ hermitienne, $L^{2}(E)$ l'espace de Hilbert associé et $\nabla^{E}$ une connection. L'application suivante

$$
\begin{aligned}
\left.\left.C^{\infty}(] 0,1\right], C^{\infty}(\widetilde{E})\right) & \longrightarrow C^{\infty}\left(E_{[] 0,1] \times N}\right), \\
\theta & \longmapsto \bar{\theta}
\end{aligned}
$$

où $\bar{\theta}(r, x):=\tau_{(1, x) \rightarrow(r, x)} \theta(r, x) \in E_{(r, x)}$ et $\tau$ est le transport parallèle le long des géodésiques radiales, permet une seconde identification :

$$
\begin{aligned}
\left.\left.U: C^{\infty}(] 0,1\right], C^{\infty}(\widetilde{E})\right) & \longrightarrow C^{\infty}(E), \\
\theta & \longmapsto r^{-\frac{1}{2} n} \bar{\theta} .
\end{aligned}
$$

L'application $U$ se prolonge en une isométrie $U: \mathcal{H} \stackrel{\simeq}{\longrightarrow} L^{2}(E)$, où $\mathcal{H}$ est le complété de $C_{c}^{\infty}(E)$ pour la norme

$$
\left(\int_{M}|\phi|^{2} \mathrm{~d} \mathrm{vol}_{M}+\int_{] 0,1]} \mathrm{d} r \int_{N}|\phi(r, x)|_{(1, x)}^{2} \mathrm{~d} \mu_{N}\right)^{\frac{1}{2}} .
$$

L'espace $\mathcal{H}=\mathcal{H}^{0,0}(E)$ sera la référence des espaces de Sobolev à poids.

\subsection{Espaces de fonctions et de sections}

DÉfinition 3.1. - On appelle $C^{k, \gamma}(E)$ l'espace des sections $C^{k}$ du fibré $E$ telles que pour tout $(i, j) \in \mathbb{N}^{2}$ vérifiant $i+j \leq k$, tout $A \in \operatorname{Diff}^{j}(N, \widetilde{E})$ on ait :

$$
p_{i, j, \gamma, A}(f)=\sup _{(r, x) \in] 0,1] \times N}\left|\left(r \partial_{r}\right)^{i} A r^{-\gamma} f(r, x)\right|_{(1, x)}<\infty .
$$

Le choix d'un recouvrement de $X$ par des ouverts trivialisants pour $E$ et les semi-normes $p_{i, j, \gamma, A}$ permettent de construire une norme $|\cdot|_{k, \gamma}$ sur $C^{k, \gamma}(E)$ qui en fait un espace de Banach. L'espace de Fréchet $\bigcap_{n>0} C^{n, \gamma}(E)$ sera noté $C^{\infty, \gamma}(E)$. Si $E=X \times \mathbb{C}$, on notera $C^{k, \gamma}(X):=C^{\infty, \gamma}(X \times \mathbb{C})$ et c'est une algèbre de Banach si $\gamma \geq 0$.

3.4. Espaces de Sobolev. — Sur les secteurs $] 0,1] \times U_{j}$, nous posons :

$$
\left(\|h\|_{s, \gamma}^{(j)}\right)^{2}=\int_{\mathbb{R}_{+}^{*} \times \mathbb{R}^{n}}\left(1+(\log \nu)^{2}+\xi^{2}\right)^{s}\left|\mathcal{F}\left(r^{-\gamma+\frac{1}{2}} h\right)(\nu, \xi)\right|^{2} \frac{\mathrm{d} \nu}{\nu} \mathrm{d} \xi,
$$

où $\mathcal{F}$ est la transformation de Fourier du groupe $\left(\mathbb{R}_{+}^{*}, \cdot\right) \times\left(\mathbb{R}^{n},+\right)$. On note $\|\cdot\|_{s}$ la norme de l'espace de Sobolev usuel $H^{s}(\bar{X})$. Puis on pose :

$$
\|f\|_{s, \gamma}^{2}=\|\varphi f\|_{s}^{2}+\sum_{j}\left(\left\|(1-\varphi) \theta_{j} f\right\|_{s, \gamma}^{(j)}\right)^{2} .
$$

Pour les fibrés hermitiens $(E, h)$, on utilise des ouverts $V_{j}$ trivialisants pour $\widetilde{E}$ sur lesquels on fixe des bases orthonormées locales. Alors pour une section 
$\left.\left.\left(h_{1}, h_{2}, \ldots, h_{k}\right)=h \in C_{c}^{\infty}(] 0,1\right], C^{\infty}(\widetilde{E})\right)$ exprimée dans cette base locale audessus de $] 0,1] \times V_{j}$, on pose :

$$
\left(\|h\|_{s, \gamma}^{(j)}\right)^{2}:=\sum_{i=1}^{k}\left(\left\|h_{i}\right\|_{s, \gamma}^{(j)}\right)^{2} .
$$

D'autres choix donnent des normes équivalentes.

DÉfinition 3.2. - L'espace de Sobolev d'ordre $s$ et de poids $\gamma$ est défini par

$$
\mathcal{H}^{s, \gamma}(E)=\left\{f \in H_{\mathrm{loc}}^{s}(E) ;\|f\|_{s, \gamma}<\infty\right\} .
$$

Cette présentation de $\mathcal{H}^{s, \gamma}$, équivalente à celles de B.W. Schulze [27] et de M. Lesch [22], entraîne immédiatement :

Proposition 3.3. - Soit $k \in \mathbb{N}$. Pour $s>k+\frac{1}{2} m$, on a une inclusion topologique

$$
\mathcal{H}^{s, \gamma}(E) \longleftrightarrow C^{k, \gamma-\frac{1}{2}}(E) .
$$

Démonstration. - Fixons $s$ et $k$ tels que $s>k+\frac{1}{2} m$ et supposons que : $E=X \times \mathbb{C}$. Soient $\phi \in C_{c}^{\infty}(X)$ et ||$_{k}$ une norme de Banach sur $C^{k}(X)$. Alors par le plongement de Sobolev usuel, on a :

$$
\forall f \in \mathcal{H}^{s, \gamma}(X), \quad \phi f \in C^{k}(X) \text { et }|\phi f|_{k} \leq c\|\phi f\|_{s} .
$$

On peut maintenant supposer que $f$ est à support dans un secteur. Par inversion de Fourier, on a pour tout $j+|\alpha| \leq k$ :

$$
\begin{aligned}
& \left|\left(r \partial_{r}\right)^{j} \partial_{x}^{\alpha} r^{-\gamma+\frac{1}{2}} f(r, x)\right| \\
& =\left|\int_{\mathbb{R}_{+}^{*} \times \mathbb{R}^{n}} \mathrm{e}^{i(\log r \cdot \log \nu+x \cdot \xi)}(i \log \nu)^{j}(i \xi)^{\alpha} \mathcal{F}\left(r^{-\gamma+\frac{1}{2}} f\right)(\nu, \xi) \frac{\mathrm{d} \nu}{\nu} \mathrm{d} \xi\right| \\
& =\mid \int_{\mathbb{R}_{+}^{*} \times \mathbb{R}^{n}} \mathrm{e}^{i(\log r \cdot \log \nu+x \cdot \xi)}(i \log \nu)^{j}(i \xi)^{\alpha}\left(1+\log ^{2} \nu+\xi^{2}\right)^{-\frac{1}{2} s} \\
& \quad \times\left(1+\log ^{2} \nu+\xi^{2}\right)^{\frac{1}{2} s} \mathcal{F}\left(r^{-\gamma+\frac{1}{2}} f\right)(\nu, \xi) \frac{\mathrm{d} \nu}{\nu} \mathrm{d} \xi \mid \\
& \leq c\|f\|_{s, \gamma}\left(\int_{\mathbb{R}_{+}^{*} \times \mathbb{R}^{n}} \log ^{2 j} \nu \xi^{2 \alpha}\left(1+\log ^{2} \nu+\xi^{2}\right)^{-s} \frac{\mathrm{d} \nu}{\nu} \mathrm{d} \xi\right)^{\frac{1}{2}} .
\end{aligned}
$$

La dernière ligne utilise l'inégalité de Cauchy-Schwarz et la dernière intégrale est bien convergente puisque $s>k+\frac{1}{2} m$. D'où :

$$
f \in C^{k, \gamma-\frac{1}{2}}(X) \text { et } \quad|f|_{k, \gamma-\frac{1}{2}} \leq c\|f\|_{s, \gamma} .
$$

Pour un fibré $E$ quelconque, on exprime la section $f$ dans une base orthonormée locale au-dessus du secteur considéré : $f=\left(f_{1}, \ldots, f_{k}\right)$, puis

$$
\left|\left(r \partial_{r}\right)^{k} \partial_{x}^{\alpha} r^{-\gamma+\frac{1}{2}} f(r, x)\right|^{2}=\sum_{i=1}^{k}\left|\left(r \partial_{r}\right)^{k} \partial_{x}^{\alpha} r^{-\gamma+\frac{1}{2}} f_{i}(r, x)\right|^{2}
$$

TOME $129-2001-\mathrm{N}^{\mathrm{O}} 4$ 
et on effectue le calcul précédent pour chaque $f_{i}$.

3.5. Opérateurs différentiels de type Fuchs. - Les opérateurs différentiels $D: C^{\infty}(E) \rightarrow C^{\infty}(E)$ de la forme suivante près de $r=0$

$$
U^{-1} D U=r^{-\nu} \sum_{k=0}^{d} a_{k}(r)\left(-r \partial_{r}\right)^{k},
$$

où $U$ est défini dans $(4), \nu \in \mathbb{R}$ et $a_{k} \in C^{\infty}\left([0,1], \operatorname{Diff}^{d-k}\left(N,\left.E\right|_{N}\right)\right)$ sont appelés opérateurs différentiels de Fuchs (voir [27], [22]) et l'espace de ces opérateurs est noté $\operatorname{Diff}^{d, \nu}(E)$. Ils sont dits elliptiques si ils sont elliptiques au sens usuel et leur symbole de Mellin $\sigma_{M}(D)=\sum_{k=0}^{d} a_{k}(0) z^{k}$ est une famille elliptique paramétrée par $\operatorname{Im} z$. Nous renvoyons aux travaux de B.W. Schulze [27], de M. Lesch [22] et de R. Melrose [24] pour l'analyse complète et détaillée de ces opérateurs.

Les opérateurs de type Dirac fournissent des exemples fondamentaux. Supposons $X$ de dimension paire et munie d'une structure spin. Le fibré des spineurs $\mathcal{S}=\mathcal{S}_{+} \oplus \mathcal{S}_{-}$(complexe, $\mathbb{Z}_{2}$-gradué) a une connection et une métrique hermitienne canoniques. Soit $W$ un fibré pourvu d'une métrique indépendante de $r$ sur la partie conique et d'une connection $\nabla^{\mathcal{W}}$ plate dans la direction radiale, i.e., de la forme

$$
\nabla^{\mathcal{W}}=\partial_{r} \otimes \mathrm{d} r+\nabla^{\widetilde{\mathcal{W}}}
$$

où $\nabla^{\widetilde{\mathcal{W}}}$ est une connection sur $\widetilde{\mathcal{W}}$. On dispose alors d'une connection de Clifford $\nabla^{\mathcal{E}}$ sur le fibré de Clifford $\mathbb{Z}_{2}$-gradué $\mathcal{E}_{+} \oplus \mathcal{E}_{-}=\mathcal{S} \otimes \mathcal{W}$. Notons $D$ l'opérateur de Dirac correspondant.

Proposition 3.4. - Sous les hypothèses précédentes, l'opérateur D appartient à $\operatorname{Diff}^{1,1}(\mathcal{E})$ et

$$
\left.\left.\forall \theta \in C^{\infty}(] 0,1\right] ; C^{\infty}(\widetilde{\mathcal{E}})\right), \quad U^{-1} D U \theta=\frac{\partial}{\partial r} \cdot \frac{\partial \theta}{\partial r}+\frac{1}{r} \widetilde{D} \theta
$$

où $\cdot$ est la multiplication de Clifford. Si on identifie $\widetilde{\mathcal{E}}$ et $\widetilde{\mathcal{E}}_{+} \oplus \partial / \partial r \cdot \widetilde{\mathcal{E}}_{+}$, l'opérateur $\widetilde{D}$ est de la forme

$$
\widetilde{D}=\left(\begin{array}{cc}
0 & D_{N} \\
D_{N} & 0
\end{array}\right)
$$

où $D_{N}$ est un opérateur de type Dirac.

REMARQUe 3.5. - L'énoncé et la preuve de cette proposition sont identiques sans structure spin : il suffit de procéder comme dans [2] pour définir les connections de Clifford et les opérateurs de type Dirac. La proposition 3.4 était connue pour l'opérateur de Dirac associé à une structure spin et sans fibré de torsion (voir [10], [21]). 
Démonstration. - Notons $e_{0}=\partial / \partial r$. Soient $\left(\tilde{e}_{1}, \ldots, \tilde{e}_{n}\right)$ une base orthonormée locale de $T N$ et $\left(e_{0}, e_{1}, \ldots, e_{n}\right)$ la base orthonormée locale de $\left.T(10,1] \times N\right)$ obtenue par transport parallèle le long des géodésiques radiales (c'est-à-dire $\left.e_{i}=\overline{\tilde{e}}_{i}\right)$. La connection canonique du fibré des spineurs est donnée par

$$
\nabla_{e_{i}}^{\mathcal{S}}=\partial_{e_{i}}+\frac{1}{2} \sum_{j<k} \omega_{j, k}\left(e_{i}\right) e_{j} \cdot e_{k},
$$

où $\omega$ est la 1-forme de la connection de Levi-Civita de (] $\left.0,1] \times N, \mathrm{~d} r^{2}+r^{2} g_{N}\right)$. Pour une section locale $\left.\left.s \in C^{\infty}(] 0,1\right] ; C^{\infty}(\widetilde{\mathcal{S}})\right)$, on obtient les relations :

avec

$$
\nabla_{e_{0}}^{\mathcal{S}} \bar{s}=\frac{\overline{\partial s}}{\partial r} \quad \text { et pour } j>0, \quad \nabla_{e_{j}}^{\mathcal{S}} \bar{s}=\frac{1}{2 r} e_{0} \cdot e_{j} \cdot \bar{s}+\frac{1}{r} \overline{\nabla_{\tilde{e}_{j}}} s,
$$

$$
\nabla_{\tilde{e}_{i}}^{\widetilde{\mathcal{S}}}=\partial_{\tilde{e}_{i}}+\frac{1}{2} \sum_{j<k} \widetilde{\omega}_{j, k}\left(\tilde{e}_{i}\right) \tilde{e}_{j} \cdot \tilde{e}_{k} .
$$

D'autre part, les conditions sur la connection de $\mathcal{W}$ entraînent les relations

$$
\nabla_{e_{0}}^{\mathcal{W}} \bar{w}=\frac{\overline{\partial w}}{\partial r} \text { et pour } j>0, \quad \nabla_{e_{j}}^{\mathcal{W}} \bar{w}=\frac{1}{r} \overline{\nabla_{\tilde{e}_{j}}^{\widetilde{\mathcal{N}}} w}
$$

Considérons maintenant la connection $\nabla^{\widetilde{\mathcal{E}}}=\nabla^{\widetilde{\mathcal{S}}} \otimes 1+1 \otimes \nabla^{\widetilde{\mathcal{W}}}$ et définissons l'opérateur $\widetilde{D}$ agissant sur les sections de $\widetilde{\mathcal{E}}$ par

$$
\widetilde{D} \theta=\sum_{j=1}^{n} \tilde{e}_{j} \nabla_{\tilde{e}_{j}}^{\widetilde{\mathcal{E}}} \theta .
$$

On obtient :

$$
\begin{aligned}
D U \theta & =e_{0} \cdot \nabla_{e_{0}}^{\mathcal{E}} r^{-\frac{1}{2} n} \bar{\theta}+\sum_{j=1}^{n} e_{j} \cdot \nabla_{e_{j}}^{\mathcal{E}} r^{-\frac{1}{2} n} \bar{\theta} \\
& =r^{-\frac{1}{2} n}\left(-\frac{n}{2 r} e_{0} \bar{\theta}+e_{0} \frac{\overline{\partial \theta}}{\partial r}+\frac{1}{r} \sum_{j=1}^{n}\left[\frac{1}{2} e_{j} \cdot e_{0} \cdot e_{j} \bar{\theta}+\overline{\tilde{e}_{j} \nabla_{\tilde{e}_{j}}^{\mathcal{E}}}\right]\right) \\
& =r^{-\frac{1}{2} n}\left(e_{0} \frac{\overline{\partial \theta}}{\partial r}+\frac{1}{r} \overline{\widetilde{D} \theta}\right)=U\left(e_{0} \frac{\partial \theta}{\partial r}+\frac{1}{r} \widetilde{D} \theta\right) .
\end{aligned}
$$

De plus, pour $\theta=\varphi+e_{0} \psi \in C^{\infty}(\widetilde{\mathcal{E}}) \simeq C^{\infty}\left(\widetilde{\mathcal{E}}_{+}\right) \oplus e_{0} C^{\infty}\left(\widetilde{\mathcal{E}}_{+}\right)$, on obtient :

$$
\begin{aligned}
\widetilde{D}\left(\varphi+e_{0} \psi\right) & =\sum_{j=1}^{n} \tilde{e}_{j} \nabla_{\tilde{e}_{j}}^{\widetilde{\mathcal{E}}}\left(\varphi+e_{0} \psi\right)=\sum_{j=1}^{n} \tilde{e}_{j}\left(\nabla_{\tilde{e}_{j}}^{\widetilde{\mathcal{E}}} \varphi+e_{0} \nabla_{\tilde{e}_{j}}^{\widetilde{\mathcal{E}}} \psi\right) \\
& =\sum_{j=1}^{n} e_{0} \tilde{e}_{j} e_{0} \nabla_{\widetilde{e}_{j}}^{\widetilde{\mathcal{E}}} \varphi+\tilde{e}_{j} e_{0} \nabla_{\tilde{e}_{j}}^{\widetilde{\mathcal{E}}} \psi \\
& =e_{0} D_{N} \varphi+D_{N} \psi,
\end{aligned}
$$

TOME $129-2001-\mathrm{N}^{\mathrm{O}} 4$ 
où on a posé

$$
D_{N}=\sum_{j=1}^{n} \tilde{e}_{j} e_{0} \nabla_{\tilde{e}_{j}}^{\widetilde{\mathcal{E}}} .
$$

Par construction, $\widetilde{\mathcal{S}_{+}}$et $\widetilde{\mathcal{S}_{-}}$sont les deux fibrés de Clifford irréductibles non équivalents sur $N$ et $\widetilde{\mathcal{S}_{-}}=e_{0} \widetilde{\mathcal{S}_{+}}$. Rappelons que l'action de $\mathrm{Cl}(N)$ (le fibré en algèbres de Clifford au-dessus de $N$, voir [20]) sur ces fibrés provient de l'action de l'algèbre de Clifford $\mathrm{Cl}^{0}(X)$ via l'application

$$
T_{p} N \ni X \longmapsto X e_{0} \in \mathrm{Cl}^{0}(X)_{\mid}(1, p) \text {. }
$$

Alors, $D_{N}$ est l'opérateur de Dirac sur le fibré de Clifford $\widetilde{\mathcal{E}_{+}}=\widetilde{\mathcal{S}_{+}} \otimes \widetilde{\mathcal{W}}$, équipé de la restriction de la connection de Clifford $\nabla^{\widetilde{\mathcal{E}}}$.

REMARQue 3.6. - En dimension impaire, on obtient :

$$
U^{-1} D U=e_{0} \partial_{r}+\frac{1}{r} D_{N}
$$

3.6. L'algèbre $\mathcal{A}$. — Définissons $\mathcal{A}=C_{c}^{\infty}(X) \oplus \mathbb{C}$ et $\mathcal{A}_{\alpha}:=C^{\infty, \alpha}(X) \oplus \mathbb{C}$ pour tout $\alpha>0$. La proposition suivante est une application directe de [3].

Proposition 3.7. - Pour tout $\alpha>0$, les sous-algèbres $\mathcal{A}$ et $\mathcal{A}_{\alpha} \operatorname{de} C\left(X^{c}\right)$ ont la même $K$-théorie que $C\left(X^{c}\right)$ :

$$
K^{j}\left(X^{c}\right)=K_{j}\left(C\left(X^{c}\right)\right)=K_{j}(\mathcal{A})=K_{j}\left(\mathcal{A}_{\alpha}\right), \quad j=0,1 .
$$

Les résultats en cohomologie cyclique sont similaires :

Proposition 3.8. - On a canoniquement

$$
H P^{*}(\mathcal{A}) \simeq H_{*}\left(X^{c} ; \mathbb{C}\right),
$$

où $H_{*}$ désigne l'homologie singulière avec sa $Z_{2}$ graduation et $H P^{*}$ est la cohomologie cyclique périodique continue.

Démonstration. — On a

$$
H P^{0}(\mathcal{A})=H P^{0}\left(C_{c}^{\infty}(X)\right) \oplus \mathbb{C} \quad \text { et } H P^{1}(\mathcal{A})=H P^{*}\left(C_{c}^{\infty}(X)\right) .
$$

Comme $C_{c}^{\infty}(X)$ est $H$-unitaire, son homologie de Hochschild est celle du complexe $\left(C_{*}\left(C_{c}^{\infty}(X)\right), b\right)$ qui se calcule comme dans [11] avec la même résolution projective. A. Connes considérait des variétés compactes, mais sa méthode s'applique sans modification au cas des fonctions $C^{\infty}$ à support compact d'une variété paracompacte. D'où :

$$
H H^{*}\left(C_{c}^{\infty}(X)\right) \simeq \operatorname{hom}_{\text {cont }}\left(\Omega_{c}^{*}(X), \mathbb{C}\right)=\mathcal{D}_{*}^{\mathcal{F}}(X) .
$$

Ici $\mathcal{D}_{*}^{\mathcal{F}}(X)$ désigne les courants de de Rham sur $X$ et l'isomorphisme est canonique. Le bord $B$ correspond au bord des courants, donc :

$$
H P^{*}\left(C_{c}^{\infty}(X)\right) \simeq H_{*}\left(\mathcal{D}^{\mathcal{F}}, \partial\right)
$$

BULletin DE LA SOCIÉtÉ MATHÉMATIQUE DE FRANCE 
L'homologie des courants donne l'homologie à support fermé de $X$ [26] qui est canoniquement isomorphe à l'homologie réduite de $X^{c}$. On obtient donc :

$$
H P^{*}(\mathcal{A} / \mathbb{C}) \simeq \bar{H}_{*}\left(X^{c} ; \mathbb{C}\right) \quad \text { et } \quad H P^{*}(\mathcal{A}) \simeq H_{*}\left(X^{c} ; \mathbb{C}\right)
$$

Des difficultés surgissent pour l'homologie de Hochschild et l'homologie cyclique des algèbres $A_{\alpha}$ (elles ne sont pas $H$-unitaires), mais la cohomologie périodique étant invariante par homotopie et excisive, on obtient :

Proposition 3.9. - Pour tout $\alpha \in] 0, \infty]$, on $a$ :

$$
H P^{*}\left(\mathcal{A}_{\alpha}\right) \simeq H P^{*}(\mathcal{A})
$$

Preuve de la proposition. - Considérons pour tout $t \in[0,1]$ les morphismes $\left.\left.\left.\left.\rho_{t}: C^{\infty, \alpha}(] 0,1\right] \times N\right) \rightarrow C^{\infty, \alpha}(] 0,1\right] \times N\right)$ définis par

$$
\rho_{t} f(r, x)=f(t r, x) \text {. }
$$

En cohomologie cyclique périodique, il vient $0=\rho_{0}^{*}=\rho_{1}^{*}=\mathrm{id}$ donc

$$
\left.\left.H P^{*}\left(C^{\infty, \alpha}(] 0,1\right] \times N\right)\right)=0
$$

et de même $\left.\left.H P^{*}\left(C_{c}^{\infty}(] 0,1\right] \times N\right)\right)=0$.

Considérons le diagramme commutatif suivant, dont les lignes sont exactes :

$$
\begin{array}{ccc}
C^{\infty, \alpha}(X) & \left.\left.\stackrel{r_{1} \times-r_{2}}{\longrightarrow} C^{\infty, \alpha}(] 0,1\right] \times N\right) \times C^{\infty}\left(X_{\frac{1}{2}}\right) \stackrel{s_{1} \times s_{2}}{\longrightarrow} C^{\infty}\left(\left[\frac{1}{2}, 1\right] \times N\right) \\
\uparrow i & \uparrow i \times \mathrm{id} & \uparrow \mathrm{id} \\
C_{c}^{\infty}(X) & \left.\left.\stackrel{r_{1} \times-r_{2}}{\longrightarrow} C_{c}^{\infty}(] 0,1\right] \times N\right) \times C^{\infty}\left(X_{\frac{1}{2}}\right) \stackrel{s_{1} \times s_{2}}{\longrightarrow} C^{\infty}\left(\left[\frac{1}{2}, 1\right] \times N\right)
\end{array}
$$

où $X_{\frac{1}{2}}=\left[\frac{1}{2}, 1\right] \times N \cup M, i$ est l'inclusion et $r_{j}, s_{j}, j=1,2$ sont les restrictions. En tenant compte des isomorphismes

$$
\begin{gathered}
H P^{*}(A \times B) \simeq H P^{*}(A) \oplus H P^{*}(B), \\
H P^{*}\left(C^{\infty}\left(X_{\frac{1}{2}}\right)\right) \simeq H P^{*}\left(C^{\infty}(M)\right), \\
H P^{*}\left(C^{\infty}\left(\left[\frac{1}{2}, 1\right] \times N\right) \simeq H P^{*}\left(C^{\infty}(N)\right),\right.
\end{gathered}
$$

on obtient :

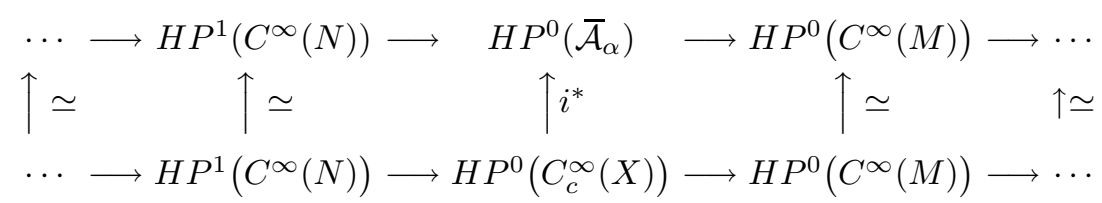

Donc $i^{*}$ est un isomorphisme et la proposition est prouvée.

TOME $129-2001-\mathrm{N}^{\mathrm{O}} 4$ 
Remarque 3.10. - Pour la version algébrique, on utilise les suites exactes :

$$
(\forall \beta<\alpha<\infty) \quad 0 \rightarrow C^{\infty, \alpha}(X) \longrightarrow C^{\infty, \beta}(X) \longrightarrow C^{\infty, \beta}(X) / C^{\infty, \alpha}(X) \rightarrow 0 .
$$

Le quotient $C^{\infty, \beta}(X) / C^{\infty, \alpha}(X)$ est nilpotent donc sa cohomologie périodique est nulle et comme $H P_{\text {alg }}^{*}$ est excisive (voir [15]), on en déduit que :

$$
H P_{\text {alg }}^{*}\left(\mathcal{A}_{\alpha}\right) \simeq H P_{\text {alg }}^{*}\left(\mathcal{A}_{\beta}\right)
$$

Toutes ces algèbres sont de bons réceptacles pour le caractère de Chern

$$
\mathrm{Ch}^{*}: K_{*}\left(X^{c}\right) \longrightarrow H_{*}\left(X^{c}, \mathbb{Q}\right)
$$

et nous choisirons la plus petite d'entre elles, en l'occurrence $\mathcal{A}$, pour simplifier les calculs.

\section{Exemples de triplets spectraux}

4.1. Énoncé du résultat. — Nous allons construire des triplets spectraux à partir d'extensions auto-adjointes $\mathcal{D}$ d'opérateurs différentiels $D$ de Fuchs elliptiques (symétriques) d'ordre 1, agissant sur les sections d'un fibré $E$. J. Brüning et R. Seeley [6], puis M. Lesch [22] ont étudié leur propriété Fredholm et dans de nombreux cas obtenu le développement asymptotique de leur noyau de la chaleur. En particulier, $\mathcal{D}$ est discret, à résolvante $(m+1)$-sommable, $m$ étant la dimension de $X$. On fixe une décomposition spectrale $\left(\phi_{k}, \lambda_{k}\right)_{k}$ de $\mathcal{D}$. Nous nous limiterons aux cas $(\imath),(\imath \imath)$ et $(\imath \imath \imath)$ décrits ci-dessous.

(i) Si $D$ est essentiellement auto-adjoint alors $\mathcal{D}$, son extension auto-adjointe est permise.

(ii) Si les coefficients $a_{k}$ de $D$ sont constants près de la singularité, alors toutes les extensions $\mathcal{D}$ de $D \in \operatorname{Diff}^{1, \nu}(E)$ correspondant aux extensions $\mathcal{D}_{0}$ de l'opérateur modèle $D_{0}$ sur le cylindre $\mathbb{R}_{+} \times N$ (déduit de $D$ de façon évidente) et vérifiant la condition ci-dessous (voir [22], p. 80) sont permises :

$$
\left(V_{t}\right)^{*} \mathcal{D}_{0} V_{t}=t^{\nu} P_{0}
$$

où $V_{t}$ est l'isométrie de $\mathcal{H}^{0,0}\left(\mathbb{R}_{+} \times N, E\right)$ telle que $V_{t}(f)(r, x)=t^{\frac{1}{2}} f(t r, x)$.

(iii) Si $E$ est $\mathbb{Z}_{2}$ gradué et si $D$ est de degré 1 par rapport à cette graduation :

$$
D=\left(\begin{array}{cc}
0 & P^{t} \\
P & 0
\end{array}\right)
$$

alors les extensions auto-adjointes

$$
\mathcal{D}^{\prime}=\left(\begin{array}{cc}
0 & P_{\max }^{t} \\
P_{\min } & 0
\end{array}\right), \quad \mathcal{D}^{\prime \prime}=\left(\begin{array}{cc}
0 & P_{\min }^{t} \\
P_{\max } & 0
\end{array}\right) \quad \text { et } \quad \mathcal{D}_{\gamma}
$$

où $\mathcal{D}_{\gamma}$ est l'extension associée à l'isométrie (induite par l'opérateur de graduation) $\gamma: \operatorname{ker}\left(D^{*}+i\right) \longrightarrow \operatorname{ker}\left(D^{*}-i\right)$, sont permises. 
L'espace de Hilbert sera $\mathcal{H}=\mathcal{H}^{0,0}(E)(c f . \S 3.4)$ et l'algèbre $\mathcal{A}$ a déjà été choisie au $\S$ 3.6. On peut maintenant énoncer :

THÉORÈme 4.1. - Sous les hypothèses précédentes, $(\mathcal{A}, \mathcal{H}, \mathcal{D})$ est un triplet spectral. Le spectre des dimensions est :

$$
S d=\{m-n ; n \in \mathbb{N}\},
$$

la multiplicité est inférieure ou égale à 2.

Schéma de la preuve. - L'hypothèse $\left(C_{1}\right)$ sera vérifiée directement. Pour $\left(C_{2}\right)$, nous allons décomposer $\varphi|\mathcal{D}|^{z}$, pour $\varphi \in C_{c}^{\infty}(X)$, comme la somme d'un opérateur pseudo-différentiel à support compact et d'un opérateur à noyau controlé. Cette décomposition est stable après composition par des opérateurs pseudodifférentiels et le prolongement méromorphe en découlera. L'approche de [4] aurait pu aussi être utilisée pour traiter cette partie.

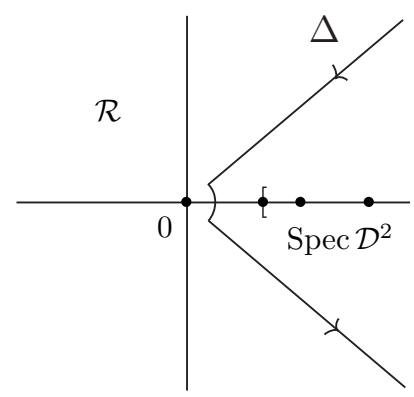

Figure 2. Le secteur $\mathcal{R}$

4.2. Préparation technique. - Nous aurons besoin d'opérateurs pseudodifférentiels à paramètre (voir [28]). Le lieu des paramètres est la région fermée $\mathcal{R}$ délimitée par $\Delta$, voir la figure 2 .

$\Delta$ est donc à distance $d>0$ du spectre de $\mathcal{D}^{2}$. Notons $\Psi_{c}^{*}(E)\left(\operatorname{resp} . \Psi_{c}^{*}(E, \mathcal{R})\right)$ l'algèbre des opérateurs pseudo-différentiels classiques (resp. classiques à paramètre) dont le noyau de Schwartz est à support compact dans $X \times X$ (resp. à support contenu dans un compact de $X \times X$ indépendant du paramètre). $\Delta$ peut être utilisé pour définir $|\mathcal{D}|^{z}$, que $\mathcal{D}$ soit inversible ou non, par

$$
|\mathcal{D}|^{z}=\frac{i}{2 \pi} \int_{\Delta} \lambda^{z / 2}\left(\mathcal{D}^{2}-\lambda\right)^{-1} \mathrm{~d} \lambda .
$$

L'énoncé suivant est un exercice classique du calcul pseudo-différentiel.

TOME $129-2001-\mathrm{N}^{\mathrm{O}} 4$ 
Proposition 4.2. - Soit $\varphi \in C_{c}^{\infty}(X)$ telle que $\varphi=1$ pour $r \geq \varepsilon$. Pour tout $n_{0} \in \mathbb{N}$, on peut construire des opérateurs $B(\lambda), C(\lambda) \in \Psi_{c}^{-\overline{2}}(E, \mathcal{R})$ et $R(\lambda), S(\lambda) \in \Psi_{c}^{-n_{0}}(E, \mathcal{R})$ tels que

$$
B(\lambda)\left(D^{2}-\lambda\right)=\varphi+R(\lambda) \quad \text { et } \quad\left(D^{2}-\lambda\right) C(\lambda)=\varphi+S(\lambda) .
$$

De plus, $B(\lambda)$ et $C(\lambda)$ dépendent analytiquement en $\lambda$ sur $\mathbb{C}$ privé d'un voisinage de 0 .

Nous allons prouver :

Proposition 4.3. - Pour tout $n \in \mathbb{N}$, on peut fixer $n_{0}$ assez grand tel que, avec les notations précédentes, les opérateurs

$$
R(\lambda)\left(\mathcal{D}^{2}-\lambda\right)^{-1}, \quad\left(\mathcal{D}^{2}-\lambda\right)^{-1} S(\lambda)
$$

soient à noyaux de Schwartz dans $C^{n, \varepsilon-\frac{1}{2}}(E) \hat{\otimes}_{\pi} C^{n, \varepsilon-\frac{1}{2}}(E)$ pour un certain $\varepsilon>0$ et ils sont analytiques en $\lambda$ dans $\mathcal{R} \backslash\{z \in \mathbb{C} ;|z| \leq 1\}$.

Lemme 4.4. - Pour tout $N \in \mathbb{N}$, il existe $c_{N}$ tel que :

$$
\forall \lambda \in \operatorname{Spec} \mathcal{D}, \forall \phi \in \operatorname{ker}(\mathcal{D}-\lambda), \quad\|\phi\|_{-N,-N} \leq c_{N} \lambda^{-N} .
$$

Preuve du lemme. - Par régularité elliptique, $\phi$ est $C^{\infty}$. Comme $\mathcal{D}^{N} \phi=$ $D^{N} \phi$, on a $\lambda^{N} \phi=\mathcal{D}^{N} \phi=D^{N} \phi$. D'autre part, $D^{N}$ appartient à Diff ${ }^{N, N}(E)$ et il a des prolongements continus

$$
D^{N}: \mathcal{H}^{s, \gamma}(E) \longrightarrow \mathcal{H}^{s-N, \gamma-N}(E),
$$

pour tout $s, \gamma \in \mathbb{R}$ (voir [22], [27]). Donc pour $s=\gamma=0$,

$$
\|\phi\|_{-N,-N}=\lambda^{-N}\left\|D^{N} \phi\right\|_{-N,-N} \leq c_{N} \lambda^{-N}\|\phi\|_{0,0}=c_{N} \lambda^{-N} .
$$

Les lemmes suivants sont des applications directes de [28] et [22] :

Lemme 4.5. - Soient $N, p \in \mathbb{N}$. Pour $k=k(p, N)$ assez grand et $C(\lambda)$ dans $\Psi_{c}^{-k}(E, \mathcal{R})$, la norme de $C(\lambda): \mathcal{H}^{-N, \gamma}(E) \rightarrow \mathcal{H}^{N, \delta}(E), \gamma, \delta \in \mathbb{R}$ vérifie

$$
\|C(\lambda)\|_{(-N, \gamma) \longrightarrow(N, \delta)} \leq c(1+|\lambda|)^{-p}
$$

LEMME 4.6. - Il existe une suite $\left(\varepsilon_{j}\right)_{j}$ de réels strictement positifs tels que

$$
\forall j \in \mathbb{N}, \forall \phi \in \operatorname{Dom} \mathcal{D}^{j}, \quad\|\phi\|_{j, \varepsilon_{j}} \leq c_{j}\left(\|\phi\|_{0,0}+\left\|\mathcal{D}^{j} \phi\right\|_{0,0}\right) .
$$

En particulier, on a $\left\|\phi_{k}\right\|_{j, \varepsilon_{j}} \leq c_{j}\left(1+\left|\lambda_{k}\right|\right)^{j}$ pour tout $k$.

Une telle suite est fixée à partir de maintenant. 
Preuve de la proposition (4.3). — L'opérateur $R(\lambda)\left(\mathcal{D}^{2}-\lambda\right)^{-1}$ est donné par :

$$
\sum_{\lambda_{k} \in \operatorname{Spec} \mathcal{D}^{2}}\left(\lambda_{k}^{2}-\lambda\right)^{-1} R(\lambda)\left(\phi_{k}\right) \otimes \bar{\phi}_{k} .
$$

Notons $|\cdot|_{m, \alpha ; t, \beta}$ la norme de $C^{m, \alpha}(E) \hat{\otimes}_{\pi} C^{t, \beta}(E)$. À l'aide du plongement de Sobolev (3.3) et des lemmes (4.5), (4.4) nous obtenons, pour tout $p, n, N$, tout $\lambda_{k} \neq 0$ et pour $n_{0}(n, N)$ convenablement choisi :

$$
\begin{aligned}
\left|\left(R(\lambda)\left(\phi_{k}\right)(x) \otimes \phi_{k}(y)\right)\right|_{n, \varepsilon_{j}-\frac{1}{2} ; n, \varepsilon_{j}-\frac{1}{2}} & \leq c\left\|R(\lambda) \phi_{k}\right\|_{j, \varepsilon_{j}}\left\|\phi_{k}\right\|_{j, \varepsilon_{j}} \\
& \leq c \frac{\left(1+\left|\lambda_{k}\right|\right)^{j}}{(1+|\lambda|)^{p}}\left\|\phi_{k}\right\|_{-N,-N} \\
& \leq c \frac{\left(1+\left|\lambda_{k}\right|\right)^{j}}{(1+|\lambda|)^{p}} \lambda_{k}^{-N} \\
& \leq c(1+|\lambda|)^{-p} \lambda_{k}^{-N+j} .
\end{aligned}
$$

La constante $c$ dépend de $p, n, N$ mais pas de $k, \lambda$. Pour $\phi_{k} \in \operatorname{ker} \mathcal{D}^{2}$, on obtient de la même façon :

$$
\left|\left(R(\lambda)\left(\phi_{k}\right)(x) \otimes \phi_{k}(y)\right)\right|_{n, \varepsilon_{j}-\frac{1}{2} ; n, \varepsilon_{j}-\frac{1}{2}} \leq c(1+|\lambda|)^{-p} .
$$

Le comportement en $\lambda$ servira ultérieurement. $\Delta$ est choisi de telle sorte que

$$
\exists M \text { tel que } \forall \lambda \in \Delta, \forall \lambda_{k} \in \operatorname{Spec} \mathcal{D}^{2}, \quad\left|\lambda_{k}^{2}-\lambda\right|^{-1} \leq M \lambda_{k}^{-2} .
$$

ker $\mathcal{D}^{2}$ est de dimension fini et inclus dans $\mathcal{H}^{q, \varepsilon_{q}}$ pour tout $q$, donc dans $C^{n, \varepsilon_{q}-\frac{1}{2}}(E)$ pour $q$ assez grand.

Ainsi, la série $(7)$ converge absolument dans $C^{n, \varepsilon-\frac{1}{2}}(E) \hat{\otimes}_{\pi} C^{n, \varepsilon-\frac{1}{2}}(E)$ dès que $-N+j-2<-1$ et la première partie est prouvée.

D'autre part, $R(\lambda)\left(\mathcal{D}^{2}-\lambda\right)^{-1}$ est analytique en $\lambda$ dans la région requise car, par construction, $R(\lambda)$ et $\left(\mathcal{D}^{2}-\lambda\right)^{-1}$ le sont. Le raisonnement est identique pour le second opérateur de la proposition 4.3 .

Proposition 4.7. - a) Pour tout $M \in \mathbb{R}$, tout $n \in \mathbb{N}$ et pour $n_{0}=n_{0}(n, M)$ assez grand, l'intégrale

$$
R_{z}=\int_{\Delta} \lambda^{z / 2} R(\lambda)\left(\mathcal{D}^{2}-\lambda\right)^{-1} \mathrm{~d} \lambda
$$

converge dans $\mathcal{B}(\mathcal{H})$ pour tout $\operatorname{Re} z<M$. Le noyau de $R_{z}$ est une fonction holomorphe de $z$ à valeurs dans $C^{n, \varepsilon-\frac{1}{2}}(E) \hat{\otimes}_{\pi} C^{n, \varepsilon-\frac{1}{2}}(E)$ pour un certain $\varepsilon>0$.

b) Pour Re $z<0$ l'intégrale

$$
B_{z}=\int_{\Delta} \lambda^{z / 2} B(\lambda) \mathrm{d} \lambda
$$

TOME $129-2001-\mathrm{N}^{\mathrm{O}} 4$ 
est absolument convergente dans $\mathcal{B}(\mathcal{H})$ et $B_{z} \in \Psi_{c}^{z}(E)$. Les composantes homogènes de son symbole sont, en coordonnées locales,

$$
b_{k}^{(z), 0}(x, \xi)=\int_{\Delta} \lambda^{z / 2} b_{k}^{0}(x, \xi, \lambda) \mathrm{d} \lambda
$$

où les $b_{k}^{0}$ sont les composantes homogènes du symbole de $B(\lambda)$.

c) les résultats de a) et $\mathrm{b})$ sont valables pour les opérateurs

$$
\int_{\Delta} \lambda^{s}\left(\mathcal{D}^{2}-\lambda\right)^{-1} S(\lambda) \text { et } \int_{\Delta} \lambda^{s} C(\lambda) \mathrm{d} \lambda .
$$

REMARQUe 4.8. - On peut prolonger la définition précédente de $B_{z}$ à tout $z \in \mathbb{C}$ de telle sorte que la relation :

$$
\text { Pour tout } k \text { tel que } \operatorname{Re} z-2 k<0, \quad B_{z}=B_{z-2 k} D^{2 k},
$$

soit vérifiée au niveau symbolique et donc modulo $\Psi_{c}^{-\infty}(E)$. De plus, en coordonnées locales, les composantes homogènes du symbole de l'opérateur $B_{z}$ obtenu sont holomorphes en $z$ sur $\mathbb{C}$ pour tout $x$ et tout $\xi \neq 0$.

Preuve de la proposition. - a) Il suffit d'utiliser les estimations (12) établies dans la preuve de 4.3, d'y choisir $p, N$ assez grands pour que l'intégrale

$$
\int_{\Delta} \lambda^{z / 2}\left(\lambda_{k}-\lambda\right)^{-1} R(\lambda)\left(\phi_{k}\right) \otimes \phi_{k} \mathrm{~d} \lambda
$$

converge absolument pour la norme $|\cdot|_{n, \varepsilon-\frac{1}{2} ; n, \varepsilon-\frac{1}{2}}$ avec un contrôle de cette intégrale par une puissance de $\lambda_{k}$ telle que la série

$$
\sum_{k \in \mathbb{N}} \int_{\Delta} \lambda^{z / 2}\left(\lambda_{k}-\lambda\right)^{-1} R(\lambda)\left(\phi_{k}\right) \otimes \phi_{k} \mathrm{~d} \lambda
$$

converge avec les propriétés exigées pour sa somme.

b) Écrivons $B_{z}=\sum_{j} \int_{\Delta} \lambda^{z / 2} B_{j}(\lambda) \mathrm{d} \lambda=\sum_{j} B_{j, z}$. Chacune des intégrales définissant $B_{j, z}$ peut être faite sur un ouvert de carte et la question peut donc être traitée en coordonnées locales où le résultat est celui de [28]. L'intégrale de contour ne modifie pas les supports des noyaux des $B_{j}(\lambda)$, donc $B_{z}$ est compactement supporté, uniformément en $z$.

c) est montré par les mêmes arguments.

On utilisera la définition de $R_{z}$ donnée dans la proposition 4.7 uniquement pour $\operatorname{Re} z<0$. Pour $\operatorname{Re} z \geq 0$ on choisit $k$ tel que $\operatorname{Re} z-2 k<0$ et on définira

$$
R_{z}:=R_{z-2 k} \mathcal{D}^{2 k}
$$

Nous ferons la même chose pour $S_{z}$. En vertu de la proposition 4.3 , b), le noyau de ce nouvel opérateur a le même comportement que celui défini par l'intégrale de contour dans la proposition 4.7. 
Proposition 4.9. - Avec les notations précédentes, la relation suivante est vérifiée pour tout $z \in \mathbb{C}$ :

$$
\varphi|\mathcal{D}|^{z}=B_{z}+R_{z} \quad \text { et } \quad|\mathcal{D}|^{z} \varphi=C_{z}+S_{z} .
$$

Démonstration. - Partons de l'égalité, sur $C_{c}^{\infty}(X)$ :

$$
B(\lambda)\left(D^{2}-\lambda\right)=\varphi+R(\lambda) .
$$

Ces opérateurs, en tant qu'opérateurs pseudo-différentiels d'ordre 0 compactement supportés, se prolongent continûment à $\mathcal{H}$ (nous surlignerons les opérateurs pour désigner leur prolongement à l'espace de Sobolev approprié). Nous admettons pour le moment l'égalité dans $B(\mathcal{H})$ suivante

$$
\overline{B(\lambda)\left(D^{2}-\lambda\right)}\left(\mathcal{D}^{2}-\lambda\right)^{-1}=\overline{B(\lambda)},
$$

qui implique l'égalité

$$
\varphi\left(\mathcal{D}^{2}-\lambda\right)^{-1}=B(\lambda)-R(\lambda)\left(\mathcal{D}^{2}-\lambda\right)^{-1},
$$

dont on déduit pour tout $\operatorname{Re} z<0$ :

$$
\varphi|\mathcal{D}|^{z}=B_{z}+R_{z}
$$

Pour $\operatorname{Re} z \geq 0$, on fixe $2 k>\operatorname{Re} z$. L'égalité (14) pour la valeur $z-2 k$ composée à droite avec l'opérateur $\mathcal{D}^{2 k}$ donne :

$$
\varphi|\mathcal{D}|^{z}=\varphi|\mathcal{D}|^{z-2 k} \mathcal{D}^{2 k}=B_{z-2 k} \mathcal{D}^{2 k}+R_{z-2 k} \mathcal{D}^{2 k} .
$$

Lorsque nous justifierons (13), nous prouverons aussi l'égalité

$$
B_{z-2 k} \mathcal{D}^{2 k}=B_{z-2 k} D^{2 k} \mathcal{D}^{-2 k} \mathcal{D}^{2 k}=B_{z-2 k} D^{2 k},
$$

qui implique $B_{z-2 k} \mathcal{D}^{2 k}=B_{z}$.

De même, les relations $R_{z-2 k} \mathcal{D}^{2 k}=R_{z-2 k} D^{2 k}$ et (15) prouvent que la définition de $R_{z}$ pour $\operatorname{Re} z \geq 0$ est indépendante de $k$. Elles permettent également d'étendre la relation (14) à $z \in \mathbb{C}$.

Nous prouvons maintenant (13) et (16).

Lemme 4.10. - Soit $A \in \operatorname{Diff}^{k, \ell}(E)$ elliptique et symétrique. Soit $\bar{A}$ son prolongement continu entre $\mathcal{H}$ et $\mathcal{H}^{-k,-\ell}(E)$. Si $A_{e}$ est une extension fermée de $A$ sur $\mathcal{H}$ de domaine $\mathcal{D}_{e}$, alors :

$$
\bar{A}_{\mid \mathcal{D}_{e}}=A_{e} .
$$

Démonstration. - Il suffit de le vérifier pour l'extension maximale $A_{\text {max }}$ de $A$ sur $\mathcal{H}$. On rappelle que $u \in \operatorname{Dom} A_{\max }$ si et seulement si la forme linéaire :

$$
\begin{aligned}
C_{c}^{\infty}(E) & \longrightarrow \mathbb{C}, \\
\varphi & \longmapsto\left\langle u, A^{*} \varphi\right\rangle
\end{aligned}
$$

TOME $129-2001-\mathrm{N}^{\mathrm{O}} 4$ 
se prolonge continûment à $\mathcal{H}$. Dans ce cas $A_{\max } u$ est l'unique élément de $\mathcal{H}$ tel que

$$
\forall v \in C_{c}^{\infty}(E), \quad\left\langle A_{\max } u, v\right\rangle=\left\langle u, A^{*} v\right\rangle .
$$

D'autre part, l'opérateur borné $\bar{A}: H \longrightarrow \mathcal{H}^{-k,-\ell}(E)$ admet un adjoint

$$
\bar{A}^{*}: \mathcal{H}^{k, \ell}(E) \simeq\left(\mathcal{H}^{-k,-\ell}(E)\right)^{\prime} \longrightarrow \mathcal{H}^{\prime} \simeq \mathcal{H},
$$

caractérisé de la façon suivante sur $C_{c}^{\infty}(E)$ (dense dans $\mathcal{H}^{k, \ell}(E)$ ) :

$$
\forall v \in \mathcal{H}, \quad\left\langle\bar{A}^{*} \varphi, v\right\rangle=\langle\varphi, \bar{A} v\rangle .
$$

La forme linéaire $\bar{A}^{*} \varphi$ sur $\mathcal{H}$ est déterminée par densité sur $C_{c}^{\infty}(E)$. On a :

$$
\forall \psi \in C_{c}^{\infty}(E) \quad\left\langle\bar{A}^{*} \varphi, \psi\right\rangle=\langle\varphi, \bar{A} \psi\rangle=\langle\varphi, A \psi\rangle=\langle A \varphi, \psi\rangle .
$$

La dernière égalité provient de la symétrie de $A$ et du fait que $\varphi, \psi \in \operatorname{Dom} A$. On a prouvé que

$$
\bar{A}^{*} \varphi=A \varphi=\bar{A} \varphi
$$

pour tout $\varphi \in C_{c}^{\infty}(E)$. Maintenant, pour $u \in \operatorname{Dom} A_{\max }$, on a

$$
\forall \psi \in C_{c}^{\infty}(E), \quad\left\langle A_{\max } u, \psi\right\rangle=\langle u, A \psi\rangle=\left\langle u, \bar{A}^{*} \psi\right\rangle=\langle\bar{A} u, \psi\rangle,
$$

d'où $A_{\max } u=\bar{A} u$, et le lemme 4.10 est prouvé.

On peut définir le prolongement de $B(\lambda)\left(D^{2}-\lambda\right)$ à $\mathcal{H}$ par la composition :

$$
\mathcal{H}=\mathcal{H}^{0,0}(E) \stackrel{\overline{D^{2}-\lambda}}{\longrightarrow} \mathcal{H}^{-2,-2}(E) \stackrel{\overline{B(\lambda)}}{\longrightarrow} \mathcal{H}^{0,0}(E) .
$$

L'application du lemme 4.10 à $\mathcal{D}^{2}-\lambda$ donne :

$$
\left.\overline{B(\lambda)} \circ \overline{\left(D^{2}-\lambda\right)}\right|_{\text {Dom } \mathcal{D}^{2}}=\overline{B(\lambda)}\left(\mathcal{D}^{2}-\lambda\right),
$$

dont on déduit immédiatement l'égalité (13) :

$$
\overline{B(\lambda)} \circ \overline{\left(D^{2}-\lambda\right)} \circ\left(\mathcal{D}^{2}-\lambda\right)^{-1}=\overline{B(\lambda)} .
$$

En appliquant le lemme à $\mathcal{D}^{2}$ on obtient (16).

Corollaire 4.11. - $\varphi|D|^{z} \varphi$ est un opérateur pseudo-différentiel d'ordre $z \grave{a}$ noyau de Schwartz à support compact.

Démonstration. - On a :

$$
\begin{aligned}
\varphi|D|^{z} \varphi & =B_{z} \varphi+\int_{\Delta} \lambda^{z / 2} R(\lambda)\left(\mathcal{D}^{2}-\lambda\right)^{-1} \varphi \mathrm{d} \lambda \\
& =B_{z} \varphi+\int_{\Delta} \lambda^{z / 2} R(\lambda)\left(C(\lambda)+\left(\mathcal{D}^{2}-\lambda\right)^{-1} S(\lambda)\right) \mathrm{d} \lambda \\
& =B_{z}^{\prime}+\int_{\Delta} \lambda^{z / 2} R(\lambda)\left(\mathcal{D}^{2}-\lambda\right)^{-1} S(\lambda) \mathrm{d} \lambda .
\end{aligned}
$$

BULLETIN DE LA SOCIÉTÉ MATHÉMATIQUE DE FRANCE 
L'assertion $B_{z}^{\prime} \in \Psi_{c}^{z}(E)$ est immédiate. On peut construire les opérateurs $B, C, R, S$ à partir de la solution complète de l'équation symbolique menant à la proposition (4.2). Alors $R(\lambda)$ et $S(\lambda)$ sont régularisants et l'opérateur

$$
\int_{\Delta} \lambda^{z / 2} R(\lambda)\left(\mathcal{D}^{2}-\lambda\right)^{-1} S(\lambda) \mathrm{d} \lambda
$$

est régularisant et à noyau de Schwartz compactement supporté.

Deux derniers lemmes seront utilisés dans la preuve du théorème 4.1 :

Lemme 4.12 (voir [27]). - Soit $C \in \Psi_{c}^{d}(E)$. Pour tout $s \in \mathbb{R}, \gamma, \delta \in \mathbb{R}, C$ admet un prolongement continu :

$$
C: \mathcal{H}^{s, \gamma}(E) \longrightarrow \mathcal{H}^{s-d, \delta}(E)
$$

LEMmE 4.13. - Soient $\varepsilon>0$ et $T$ dans $C^{k, \alpha-\frac{1}{2}+\varepsilon}(E) \hat{\otimes}_{\pi} C^{\ell, \beta-\frac{1}{2}+\varepsilon}(E)$. L'opérateur intégral correspondant a un prolongement continu :

$$
T: \mathcal{H}^{-\ell,-\beta} \longrightarrow \mathcal{H}^{k, \alpha} \text {. }
$$

Démonstration. - D'après la proposition 3.3, les inclusions

$$
C^{k, \alpha-\frac{1}{2}+\varepsilon}(E) \longleftrightarrow \mathcal{H}^{k, \alpha} \quad \text { et } \quad C^{\ell, \beta-\frac{1}{2}+\varepsilon}(E) \longleftrightarrow \mathcal{H}^{\ell, \beta}
$$

sont continues, donc il en est de même pour

$$
C^{k, \alpha-\frac{1}{2}+\varepsilon}(E) \hat{\otimes}_{\pi} C^{\ell, \beta-\frac{1}{2}+\varepsilon}(E) \hookrightarrow \mathcal{H}^{k, \alpha} \hat{\otimes}_{\pi} \mathcal{H}^{\ell, \beta} .
$$

Rappelons que $\mathcal{H}^{-\ell,-\beta}=\left(\mathcal{H}^{\ell, \beta}\right)^{\prime}$ où la dualité est donnée par

$$
\langle f, g\rangle=\int_{X} \bar{f} g \mathrm{~d} \mu .
$$

On dispose donc d'un plongement (topologique) :

$$
\mathcal{H}^{k, \alpha} \hat{\otimes}_{\pi} \mathcal{H}^{\ell, \beta} \longleftrightarrow \mathcal{B}\left(\mathcal{H}^{-\ell,-\beta}, \mathcal{H}^{k, \alpha}\right),
$$

qui associe à $T$ l'opérateur intégral de noyau de Schwartz $T$.

\subsection{Preuve du théorème 4.1}

4.3.1. Vérification de la condition $\left(C_{1}\right)$. — Pour $p+q>0$, l'opérateur $\nabla^{p+q}(a)$ a un support compact. Pour $\varphi \in C_{c}^{\infty}(X)$ telle que $\varphi \nabla^{p+q}(a) \varphi=\nabla^{p+q}(a)$, on peut écrire :

$$
L^{p} R^{q}(a)=\left(C_{-p}+S_{-p}\right) \nabla^{p+q}(a)\left(B_{-q}+R_{-q}\right),
$$

où $C_{-p} \in \Psi_{c}^{-p}(E), B_{-q} \in \Psi_{c}^{-q}(E)$ et $S_{-p}, R_{-q} \in C^{n, \varepsilon-\frac{1}{2}} \hat{\otimes}_{\pi} C^{n, \varepsilon-\frac{1}{2}}$ avec $\varepsilon>0$ et $n$ arbitrairement grand. L'opérateur pseudo-différentiel compactement supporté $C_{-p} \nabla^{p+q}(a) B_{-q}$ est d'ordre 0 , donc borné sur $\mathcal{H}$. D'autre part, pour $n \geq p$, le lemme 4.13 implique la continuité de $S_{-p}: \mathcal{H}^{-p, 0} \rightarrow \mathcal{H}$, puis par composition :

$$
S_{-p} \nabla^{p+q}(a) B_{-q} \in \mathcal{B}(\mathcal{H})
$$

TOME $129-2001-\mathrm{N}^{\mathrm{O}} 4$ 
Les autres cas sont analogues.

4.3.2. Vérification de la condition $\left(C_{2}\right)$. - Pour tout $b \in \mathcal{B}$, la fonction $h_{b}: z \mapsto$ $\operatorname{Tr}\left(b|\mathcal{D}|^{-z}\right)$ est définie et holomorphe dans le demi-plan $\operatorname{Re} z>m$. Fixons $b \in \mathcal{B}$. On peut décomposer $b$ en une somme finie d'éléments de la forme :

$$
Q^{\prime}=|D|^{\ell_{1}} a_{1}|D|^{\ell_{2}} a_{2} \cdots|D|^{\ell_{j}} a_{j}|D|^{\ell_{j+1}},
$$

avec $a_{1}, \ldots, a_{j} \in C_{c}^{\infty}(X)$ et $\ell_{i} \in \mathbb{N}$. Remarquons que pour Re $z$ assez grand :

$$
\begin{aligned}
& \operatorname{Tr}\left(|D|^{\ell_{1}} a_{1}|D|^{\ell_{2}} a_{2} \cdots|D|^{\ell_{j}} a_{j}|D|^{\ell_{j+1}}|\mathcal{D}|^{-z}\right) \\
& =\operatorname{Tr}\left(a_{1}|D|^{\ell_{2}} a_{2} \cdots|D|^{\ell_{j}} a_{j}|\mathcal{D}|^{-z+\ell_{1}+\ell_{j+1}}\right) .
\end{aligned}
$$

D'après le corollaire 4.11, on a

$$
a_{1}|D|^{\ell_{2}} a_{2} \cdots|D|^{\ell_{j}} a_{j}=: Q \in \Psi_{c}^{|\ell|}(E) .
$$

Nous avons deux cas à considérer : le prolongement de $\operatorname{Tr}\left(|\mathcal{D}|^{-z}\right)$ et celui de $\operatorname{Tr}\left(Q|\mathcal{D}|^{-z+\ell}\right)$ avec $Q \in \Psi_{c}^{k}(E), k, \ell \in \mathbb{N}$.

Cas de $h_{1}(z)=\operatorname{Tr}|\mathcal{D}|^{-z}$. — Le prolongement méromorphe de $h_{1}(z)=$ $\operatorname{Tr}\left(|\mathcal{D}|^{-z}\right)$ résulte de celui du noyau de la chaleur qui est établi dans [22] :

$$
\operatorname{Tr} e^{-t \mathcal{D}^{2}} \sim_{t \rightarrow 0} \sum_{n=0}^{\infty}\left(a_{n}+\alpha_{n} \log t\right) t^{\frac{1}{2}(n-m)}+\sum_{n=0}^{\infty} \beta_{n} t^{\frac{1}{2} n}
$$

et de la formule

$$
\operatorname{Tr}|\mathcal{D}|^{-z}=\frac{1}{\Gamma(z / 2)} \int_{0}^{\infty} t^{z / 2-1} \operatorname{Tr} \mathrm{e}^{-t \mathcal{D}^{2}} \mathrm{~d} t
$$

Proposition 4.14. - La fonction $h_{1}$ est méromorphe sur $\mathbb{C}$ avec pôles au plus d'ordre 2 aux points $z_{n}=m-n, n \in \mathbb{N}$.

Cas de $h_{Q}(z)=\operatorname{Tr}\left(Q|\mathcal{D}|^{-z}\right)$ avec $Q \in \Psi_{c}^{\ell}(E)$. - Fixons $\varphi \in C_{c}^{\infty}(X)$ telle que $\varphi Q=Q \varphi=Q$. On part de la relation

$$
\begin{aligned}
Q|\mathcal{D}|^{-z} & =\int_{\Delta} \lambda^{-z / 2} Q B(\lambda) \mathrm{d} \lambda+\int_{\Delta} \lambda^{-z / 2} Q R(\lambda)\left(\mathcal{D}^{2}-\lambda\right)^{-1} \mathrm{~d} \lambda \\
& =Q B_{-z}+T_{-z}
\end{aligned}
$$

Alors :

Proposition 4.15. - Pour tout $Q \in \Psi_{c}^{\ell}(E)$ la fonction

$$
z \longmapsto \operatorname{Tr} Q B_{-z}=\int_{X} \operatorname{tr} Q B_{-z}(x, x) \mathrm{d} x,
$$

BULLETIN DE LA SOCIÉtÉ MATHÉMATIQUE DE FRANCE 
définie et holomorphe pour $\operatorname{Re} z>m+\ell$ se prolonge en une fonction méromorphe sur $\mathbb{C}$ avec des pôles simples aux points $z_{n}=m-n+\ell, n \in \mathbb{N}$. Les résidus sont donnés par

$$
\sum_{j+|\alpha|+k=n} \int_{S^{*} X} \operatorname{tr} \partial_{\nu}^{\alpha} q_{j}(x, \nu) \partial_{x}^{\alpha} b_{k}^{(-z)}(x, \nu) \mathrm{d} \nu \mathrm{d} x
$$

où $q_{j}, b_{k}^{(-z)}$ sont les composantes homogènes du symbole, respectivement, de $Q$ de degré $\ell-j$ et de $B_{-z}$ de degré $-\operatorname{Re} z-k$.

Démonstration. - Nous nous inspirons du chapitre 2 de [28]. Par construction, $B(\lambda)$ est une somme d'opérateurs définis dans des cartes locales. Il suffit donc de travailler avec l'un d'eux (on le notera toujours $B(\lambda)$ ) défini sur un ouvert $U$. Notons $b_{k}(x, \xi, \lambda)$ la composante homogène de degré $-2-k$ de son symbole. $Q B_{-z}$ est un opérateur pseudo-différentiel d'ordre $-z+\ell$, donc il a un noyau de Schwartz continu et holomorphe en $z$ lorsque $\operatorname{Re} z>m+\ell$, donné par

$$
\int_{\mathbb{R}^{m}} \mathrm{e}^{i(x-y) \cdot \xi} \sigma\left(Q B_{-z}\right)(x, \xi) \mathrm{d} \xi .
$$

Par construction également, $B_{-z}$ dépend d'un entier $n_{0}$ qui peut-être fixé arbitrairement grand et plus précisément

$$
B_{-z}=\sum_{k=0}^{n_{0}} \operatorname{op}\left(\theta(\xi) b_{k}^{(-z)}(x, \xi)\right)=\sum_{k=0}^{n_{0}} B_{k}^{(-z)}
$$

où $b_{k}^{(-z)}(x, \xi)=\int_{\Delta} \lambda^{-z / 2} b_{k}(x, \xi, \lambda) \mathrm{d} \lambda$ et $\theta$ sert à éliminer la singularité en $\xi=0$. L'opérateur $B_{k}^{(-z)}$ est d'ordre $-z-k$. Posons pour $N \in \mathbb{N}$ :

$$
\left(Q B_{k}^{(-z)}\right)^{\prime}=\sum_{|\alpha|+j=0}^{N} \mathrm{op}\left(\theta(\xi) \partial_{\xi}^{\alpha} q_{j}(x, \xi) \partial_{x}^{\alpha} b_{k}^{(-z)}(x, \xi),\right.
$$

de sorte que le reste $R_{k}^{(-z)}=\left(Q B_{k}^{(-z)}\right)^{\prime}-Q B_{k}^{(-z)}$ soit d'ordre $-z-N-1$ donc à noyau de Schwartz continu et holomorphe en $z$ dans le demi-plan $\operatorname{Re} z>m-N-1$.

La restriction à la diagonale du noyau de $\left(Q B_{k}^{(-z)}\right)^{\prime}$ se calcule explicitement. Pour Re $z$ assez grand, l'intégrale

$$
\int_{\mathbb{R}^{m}} \mathrm{e}^{i(x-y) \cdot \xi} \theta(\xi) \partial_{\xi}^{\alpha} q_{j}(x, \xi) \partial_{x}^{\alpha} b_{k}^{(-z)}(x, \xi) \mathrm{d} \xi
$$

converge si $|\alpha|+j+k+\ell \leq N$ et définit un noyau continu en $(x, y)$. L'utilisation des coordonnées polaires $(r, \nu) \in \mathbb{R}_{+} \times S^{m-1}$ et l'homogénéité de $\partial_{\xi}^{\alpha} q_{j}$ (le degré est $\ell-|\alpha|-j$ ) et de $b^{(-z), 0}$ (le degré est $-z-k$ ) donnent immédiatement pour l'intégrale (25), avec $x=y$ :

$$
\left(\int_{0}^{\infty} \theta(r) r^{\ell-j-|\alpha|-z-k+m-1} \mathrm{~d} r\right)\left(\int_{S^{m-1}} \partial_{\nu}^{\alpha} q_{j}(x, \nu) \partial_{x}^{\alpha} b_{k}^{(-z), 0}(x, \nu) \mathrm{d} \nu\right) .
$$

TOME $129-2001-\mathrm{N}^{\mathrm{O}} 4$ 
On peut fixer $\theta$ tel que $\theta=0$ sur $\left[0, \frac{1}{2}\right]$ et $\theta=1$ sur $[1, \infty[$; donc

$$
\int_{0}^{1} \theta(r) r^{\ell-j-|\alpha|-z-k+m-1} \mathrm{~d} r
$$

est entière et

$$
\int_{1}^{\infty} \theta(r) r^{\ell-j-|\alpha|-z-k+m-1} \mathrm{~d} r=\frac{1}{-\ell+j+|\alpha|+k-m+z}
$$

est méromorphe sur $\mathbb{C}$ avec un pôle simple en $z=m+\ell-(j+|\alpha|+k)$. D'où

$$
\operatorname{tr}\left(Q B_{k}^{(z)}\right)^{\prime}(x, x)=\sum_{n=k+\ell}^{N} \frac{c_{n}(x)}{z-(m-n)+\ell}+f(z, x),
$$

où $f$ holomorphe sur $\mathbb{C}$ à valeurs dans les fonctions continues (et à support compact) sur $U$. Les calculs précédents pouvant être faits pour $N$ arbitrairement grand, la preuve est finie.

REMARQue 4.16. - Ces résultats peuvent également être déduits du travail de G. Grubb et R. Seeley [17].

Pour le second opérateur de la somme dans (21), on montre :

Proposition 4.17. - Pour tout $k>0$ et $n_{0}=n_{0}(k)$ assez grand, le noyau de $T_{-z}$ est une fonction continue sur $X \times X$ et holomorphe en $z$ dans le demiplan $\operatorname{Re} z>-k$.

Démonstration. - Formellement :

$$
T_{-z}(x, y)=\int_{\Delta} \sum_{j \in \mathbb{N}} \frac{\lambda^{-z / 2}}{\lambda_{j}^{2}-\lambda} Q R(\lambda)\left(\phi_{j}\right)(x) \otimes \overline{\phi_{j}} \mathrm{~d} \lambda .
$$

Grâce aux lemmes 4.12 et 4.5 on a, pour $n_{0}=n_{0}(p, n, N, \ell)$ assez grand :

$$
\begin{aligned}
\left|Q R(\lambda)\left(\phi_{j}\right)\right|_{p, \varepsilon_{p}-\frac{1}{2}} & \leq c\left\|Q R(\lambda)\left(\phi_{j}\right)\right\|_{p^{\prime}, \varepsilon_{p}} \\
& \leq c\left\|R(\lambda)\left(\phi_{j}\right)\right\|_{p^{\prime}-l, \varepsilon_{p}} \leq c(1+|\lambda|)^{-n} \lambda_{j}^{-N},
\end{aligned}
$$

si $\lambda_{j} \neq 0$ et

$$
\left|Q R(\lambda)\left(\phi_{j}\right)\right|_{p, \varepsilon_{p}-\frac{1}{2}} \leq c(1+|\lambda|)^{-n}
$$

sinon. L'intégrale

$$
f_{j}(z, x)=\int_{\Delta} \frac{\lambda^{-z / 2}}{\lambda_{j}^{2}-\lambda} Q R(\lambda)\left(\phi_{j}\right)(x) \mathrm{d} \lambda
$$

converge absolument par rapport à la norme $|\cdot|_{p, \varepsilon_{p}-\frac{1}{2}}$ pour $\operatorname{Re} z>-2(n-1)$ fixé. On constate que la convergence est uniforme en $z$ pour la norme infinie sur 
les parties compactes $K$ de $\{\operatorname{Re} z>-2(n-1)\}$. En effet, vu les majorations faites,

$$
\sup _{z \in K}|f(z)|_{p, \varepsilon_{p}-\frac{1}{2}} \leq c \int_{\Delta}(1+|\lambda|)^{-\rho-1}|\mathrm{~d} \lambda| \leq c,
$$

où $\rho$ est la distance entre $K$ et la droite Re $z=-2(n-1)$. L'argument est le même pour l'intégrale

$$
\int_{\Delta}\left(-\frac{1}{2} \log \lambda\right) \frac{\lambda^{-z / 2}}{\lambda_{j}^{2}-\lambda} Q R(\lambda)\left(\phi_{j}\right)(x) \mathrm{d} \lambda,
$$

donc la fonction $f_{j}$ est holomorphe sur $\{\operatorname{Re} z>-2(n-1)\}$, à valeurs dans $C^{p, \varepsilon_{p}-\frac{1}{2}}(E)$.

De façon similaire, on établit que la série de terme général $f_{j}(z) \otimes \phi_{j}$ converge absolument par rapport à la norme $|\cdot|_{p, \varepsilon_{p}-\frac{1}{2}} \otimes|\cdot|_{p, \varepsilon_{p}-\frac{1}{2}}$ (pourvu que $N$ soit choisi assez grand) et uniformément en $z$ sur les parties compactes de $\{\operatorname{Re} z>-2(n-1)\}$. Le résultat étant identique pour la série des dérivées, la somme de la série est une fonction holomorphe en $z$ sur le demi-plan considéré, à valeurs dans $C^{p, \varepsilon_{p}-\frac{1}{2}}(E) \hat{\otimes}_{\pi} C^{p, \varepsilon_{p}-\frac{1}{2}}(E)$. On a également prouvé que l'on pouvait intervertir $\int_{\Delta}$ et $\sum_{j}$. Comme $n$ peut être fixé arbitrairement grand, la preuve est achevée.

Nous avons achevé la vérification de $\left(C_{2}\right)$.

\section{Calcul du caractère de Chern}

5.1. Énoncé du résultat. — Nous calculons explicitement, dans le contexte d'une singularité conique le caractère de Chern des opérateurs de Dirac tordus par des fibrés $E$ à l'aide de la formule de [14]. Nos calculs s'appliquent aux extensions $\mathcal{D}^{\prime}, \mathcal{D}^{\prime \prime}$ et $\mathcal{D}_{\gamma}$ (voir 4 ) et les résultats ne diffèrent que par une composante homologique de degré 0 .

ThÉORÈme 5.1. - Pour $n>0$, on $a$ :

$$
\varphi_{n}\left(a_{0}, \ldots, a_{n}\right)=\nu_{n} \int_{X} a_{0} \mathrm{~d} a_{1} \wedge \cdots \wedge \mathrm{d} a_{n} \wedge \hat{A}(X) \wedge \mathrm{Ch}(E) .
$$

Pour $n=0$, avec $a \in C_{c}^{\infty}(X)$ et $\lambda \in \mathbb{C}$, on $a$ :

$$
\varphi_{0}(a+\lambda)=\int_{X} a \hat{A}(X) \wedge \operatorname{Ch}(E)+\lambda \operatorname{Ind}\left(\mathcal{D}_{+}\right) .
$$

$\hat{A}(X)$ est la forme différentielle correspondant au genre $\hat{A}$ de la variété riemannienne $X$ et $\operatorname{ch}(E)$ est le caractère de Chern du fibré de $E$.

Plusieurs termes doivent être évalués dans la formule de A. Connes et H. Moscovici (2.2). Si $n$ et $q$ sont strictement positifs, alors la proposition (4.15) s'applique et les termes $\tau_{q}$, pour $n>0$ sont nuls. Pour $n>0$ et

TOME $129-2001-\mathrm{N}^{\mathrm{O}} 4$ 
$q=0$, nous effectuerons une «excision » de la singularité qui nous permettra ensuite d'utiliser le calcul de E. Getzler [16]. Nous conclurons par le cas $n=0$.

5.2. La preuve. - Nous commençons par le cas $n>0, q=0$.

Proposition 5.2. - Si $|k|>0$, alors

$$
\tau_{0}\left(\gamma a_{0}\left(\mathrm{~d} a_{1}\right)^{\left(k_{1}\right)} \ldots\left(\mathrm{d} a_{n}\right)^{\left(k_{n}\right)}|\mathcal{D}|^{-(2|k|+n)}\right)=0 .
$$

Si $k=0$, alors

$$
\tau_{0}\left(\gamma a_{0} \mathrm{~d} a_{1} \ldots \mathrm{d} a_{n}|\mathcal{D}|^{-n}\right)=\nu_{n} \int_{X} a_{0} \mathrm{~d} a_{1} \wedge \cdots \wedge \mathrm{d} a_{n} \wedge \hat{A}(X) \wedge \operatorname{ch}(E) .
$$

Nous allons utiliser, après un travail technique préalable, le calcul symbolique de Getzler [16] et la démarche de [13]. Nous avons ici :

$$
\tau_{0}\left(Q|\mathcal{D}|^{-(2|k|+n)}\right)=\operatorname{Res}_{w=2|k|+n} \operatorname{Str}\left(Q|\mathcal{D}|^{-w}\right),
$$

où $Q:=a_{0}\left(\mathrm{~d} a_{1}\right)^{\left(k_{1}\right)} \ldots\left(\mathrm{d} a_{n}\right)^{\left(k_{n}\right)}$. Le long de la diagonale, la relation asymptotique suivante est vérifiée :

$$
\operatorname{str} Q \mathrm{e}^{-t \mathcal{D}^{2}}(x) \sim^{t \rightarrow 0} \sum_{j=0}^{\infty} h_{j}(x) t^{\frac{1}{2}(j-m)},
$$

où $\sim^{t \rightarrow 0}$ fait référence à l'utilisation de la topologie $C^{\infty}$ sur les parties compactes de $X$. Le résidu recherché (28) correspond au coefficient de $t^{-\left(|k|+\frac{1}{2} n\right)}$. Plus précisément,

$$
\operatorname{Res}_{w=2|k|+n} \operatorname{Str}\left(Q|\mathcal{D}|^{-w}\right)=\frac{2}{\Gamma\left(|k|+\frac{1}{2} n\right)} \int_{X} h_{m-(2|k|+n)}(x) \mathrm{d} \mu .
$$

Fixons $\psi \in C_{c}^{\infty}(X)$ égale à 1 sur le support de $Q$. On a alors :

$$
\operatorname{Str} Q \mathrm{e}^{-t \mathcal{D}^{2}}=\operatorname{Str} Q \mathrm{e}^{-t \mathcal{D}^{2}} \psi \text {. }
$$

Déformons maintenant, sur un voisinage de la singularité $] 0, \varepsilon[\times N$ disjoint du support de $\psi$, la métrique conique en la métrique produit $\mathrm{d} r^{2}+g_{N}$. Notons $g_{1}$ cette nouvelle métrique et formons la variété riemannienne $C^{\infty}$ compacte sans bord $(\widetilde{X}, \widetilde{g})$ obtenue en doublant la variété riemannienne compacte à bord $\left(\bar{X}, g_{1}\right)$. On fait la même opération pour le fibré de Clifford $E$ et sa connection $\nabla^{E}$. Notons $\widetilde{D}$ l'opérateur de Dirac correspondant. Notons aussi

$$
\left.X_{\varepsilon}=X \backslash\right] 0, \varepsilon[\times N \quad \text { et } \quad \bar{X}=X \cup\{0\} \times N .
$$

Lemme 5.3. - Sous les hypothèses précédentes, on a l'égalité

$$
\operatorname{Str} Q \mathrm{e}^{-t \mathcal{D}^{2}} \psi=\operatorname{Str} Q \mathrm{e}^{-t \widetilde{\mathcal{D}}^{2}} \psi .
$$

BULletin DE LA SOCiÉtÉ MATHÉMATIQUE DE FRANCE 
Démonstration. - $D$ et $\widetilde{D}$ coïncident sur $X_{\varepsilon / 2}$. Les opérateurs $\widetilde{D}$ et $\widetilde{D}^{2}$ sont essentiellement auto-adjoints. Le contour $\Delta$ peut être choisi de sorte que, une fois la métrique déformée :

$$
\operatorname{Spec} \widetilde{D}^{2} \cap \Delta=\varnothing \text {. }
$$

Alors $\left(\widetilde{D}^{2}-\lambda\right)^{-1}$ est un opérateur pseudo-différentiel d'ordre -2 . De plus, pour $\varphi, \psi$ égales à 1 sur $X_{\varepsilon}$, nulles sur $] 0, \varepsilon[\times N$ et telles que $\varphi \psi=\varphi$, nous allons montrer que

$$
\varphi\left(\mathcal{D}^{2}-\lambda\right)^{-1} \psi=\varphi\left(\widetilde{D}^{2}-\lambda\right)^{-1} \psi
$$

en tant qu'opérateurs sur $C^{\infty}\left(X_{\varepsilon}, E\right)$ et modulo un opérateur régularisant dont le noyau de Schwartz est nul sur un voisinage de la diagonale.

Prouvons cette affirmation. Soient $\theta, \rho \in C_{c}^{\infty}\left(X_{\varepsilon / 2}\right)$ telles que $\varphi \theta=\varphi$, $\psi \theta=\psi$ et $\rho \psi=\psi$. Pour tout $v \in C_{c}^{\infty}(\mathcal{E}) \subset C^{\infty}(\widetilde{\mathcal{E}})$, l'équation

$$
\rho v=\left(\widetilde{D}^{2}-\lambda\right) w
$$

a une unique solution $w=\left(\widetilde{D}^{2}-\lambda\right)^{-1} \rho v$. Alors

$$
\psi v=\psi \rho v=\psi\left(\widetilde{D}^{2}-\lambda\right) \theta w+\psi\left(\widetilde{D}^{2}-\lambda\right)(1-\theta) w,
$$

et par construction $\left(\widetilde{D}^{2}-\lambda\right) \theta w=\left(D^{2}-\lambda\right) \theta w$. Pour tout $v \in C_{c}^{\infty}(\mathcal{E})$, on a :

$$
\begin{aligned}
\varphi\left(\widetilde{D}^{2}-\lambda\right)^{-1} \psi v & =\varphi\left(\widetilde{D}^{2}-\lambda\right)^{-1} \psi \rho v \\
& =\varphi\left(\widetilde{D}^{2}-\lambda\right)^{-1} \psi\left(\widetilde{D}^{2}-\lambda\right) w \\
& =\varphi w+\varphi\left(\widetilde{D}^{2}-\lambda\right)^{-1}\left[\psi, \widetilde{D}^{2}\right] w \\
& =\varphi w+\varphi\left(\widetilde{D}^{2}-\lambda\right)^{-1}\left[\psi, D^{2}\right] w
\end{aligned}
$$

où on a utilisé $\left[\psi, \widetilde{D}^{2}\right]=\left[\psi, D^{2}\right]$. D'autre part,

$$
\begin{aligned}
\varphi\left(\mathcal{D}^{2}-\lambda\right)^{-1} \psi v= & \varphi\left(\mathcal{D}^{2}-\lambda\right)^{-1} \psi\left\{\left(D^{2}-\lambda\right) \theta+\left(\widetilde{D}^{2}-\lambda\right)(1-\theta)\right\} w \\
= & \varphi\left(\mathcal{D}^{2}-\lambda\right)^{-1} \psi\left(D^{2}-\lambda\right) \theta w \\
& +\varphi\left(\mathcal{D}^{2}-\lambda\right)^{-1} \psi\left\{(1-\theta)\left(\widetilde{D}^{2}-\lambda\right)+\left[\widetilde{D}^{2}-\lambda, 1-\theta\right]\right\} w \\
= & \varphi w+\varphi\left(\mathcal{D}^{2}-\lambda\right)^{-1}\left[\psi, D^{2}\right] \theta w-\varphi\left(\mathcal{D}^{2}-\lambda\right)^{-1} \psi\left[\widetilde{D}^{2}, \theta\right] w \\
= & \varphi w+\varphi\left(\mathcal{D}^{2}-\lambda\right)^{-1}\left[\psi, D^{2}\right] w,
\end{aligned}
$$

où on a utilisé $(32),(1-\theta) \psi=0$ et $\left[\psi, D^{2}\right] \theta-\psi\left[\widetilde{D}^{2}, \theta\right]=\left[\psi, D^{2}\right]$. Par conséquent,

$$
\begin{aligned}
\varphi\left(\left(\widetilde{D}^{2}-\lambda\right)^{-1}-\right. & \left.\left(\mathcal{D}^{2}-\lambda\right)^{-1}\right) \psi \\
& =\varphi\left(\left(\widetilde{D}^{2}-\lambda\right)^{-1}-\left(\mathcal{D}^{2}-\lambda\right)^{-1}\right)\left[\psi, D^{2}\right]=: T(\lambda) .
\end{aligned}
$$

Le commutateur $\left[\psi, D^{2}\right]$ est un opérateur différentiel d'ordre 1 dont les coefficients sont à support dans $\operatorname{Suppd} \psi$, qui est d'intersection vide avec $\operatorname{Supp} \varphi$. Par conséquent, $\varphi\left(\mathcal{D}^{2}-\lambda\right)^{-1}\left[\psi, D^{2}\right]$ et $\varphi\left(\widetilde{D}^{2}-\lambda\right)^{-1}\left[\psi, D^{2}\right]$ sont des opérateurs régularisants donnés par des noyaux de Schwartz nuls sur un voisinage de la

TOME $129-2001-\mathrm{N}^{\mathrm{O}} 4$ 
diagonale de $\widetilde{X} \times \widetilde{X}$ et dont les supports sont des compacts inclus dans $X \times X$. Ils sont de classe trace (car régularisants et à support compact) et pour les raisons précédentes, leur trace est nulle.

Après l'intégrale de contour $\int_{\Delta} \mathrm{e}^{-t \lambda} T(\lambda) \mathrm{d} \lambda$, l'opérateur obtenu a encore un noyau nul sur un voisinage de la diagonale de $\widetilde{X} \times \widetilde{X}$ donc est à trace et à super trace nulle. On obtient donc $\operatorname{Str} \varphi \mathrm{e}^{-t \mathcal{D}^{2}} \psi=\operatorname{Str} \varphi \mathrm{e}^{-t \widetilde{\mathcal{D}}^{2}} \psi$ et les conclusions sont identiques si on remplace $\varphi$ par $Q$.

En définitive, lorsque $n>0$, on peut remplacer $\mathcal{D}^{2}$ par $\widetilde{D}^{2}$. On dispose alors des résultats prouvés dans [13]. Dans leur terminologie, on montre que :

Lemme 5.4. - $Q$ est d'ordre asymptotique 0 et de plus :

- si $|k|>0$, alors $\sigma_{0}(Q)=0$;

- si $k=0$, alors $\sigma_{0}(Q)=a_{0} \mathrm{~d} a_{1} \wedge \cdots \wedge \mathrm{d} a_{n}$.

Démonstration. - $D$ désignera ici $\widetilde{D}$ pour alléger les notations.

Les opérateurs $D^{2},[D, a]$ sont d'ordre asymptotique 0 , donc $Q$ aussi. On sait que (voir [13]) :

$$
\sigma_{0}\left(D^{2}\right)=-|\xi|^{2}+\frac{1}{2}\left(\nabla^{\mathcal{E}}\right)^{2}, \quad \sigma_{0}([D, a])=\mathrm{d} a .
$$

Si $A, B$ sont d'ordre asymptotique nul alors le produit de leur symbole asymptotique $a, b$ est donné par :

$$
a * b(x, \xi)=\mathrm{e}^{-1 / 4 R\left(\partial_{\xi}, \partial_{\eta}\right)} a(x, \xi) \wedge b(x, \eta)_{\mid \xi=\eta} .
$$

On calcule pour $Q=(\mathrm{d} a)^{(1)}$ :

$$
\begin{aligned}
\sigma_{0}\left(\left[D^{2},[D, a]\right]\right) & =\sigma_{0}\left(D^{2}\right) * \sigma_{0}([D, a])-\sigma_{0}([D, a]) * \sigma_{0}\left(D^{2}\right) \\
& =\left(-|\xi|^{2}+\frac{1}{2}\left(\nabla^{\mathcal{E}}\right)^{2}\right) * \mathrm{~d} a-\mathrm{d} a *\left(-|\xi|^{2}+\frac{1}{2}\left(\nabla^{\mathcal{E}}\right)^{2}\right) \\
& =\left(-|\xi|^{2}+\frac{1}{2}\left(\nabla^{\mathcal{E}}\right)^{2}\right) \wedge \mathrm{d} a-\mathrm{d} a \wedge\left(-|\xi|^{2}+\frac{1}{2}\left(\nabla^{\mathcal{E}}\right)^{2}\right) \\
& =0 .
\end{aligned}
$$

L'avant-dernière ligne résulte $\mathrm{du}$ fait que $\mathrm{d} a$ est indépendante des variables dans le cotangent. La dernière ligne est justifiée par les remarques suivantes.

Le symbole principal $-|\xi|^{2}$ de $D^{2}$ est scalaire et c'est une 0-forme, donc il commute avec la 1-forme $\mathrm{d} a$.

L'opérateur de courbure

$$
\left(\nabla^{\mathcal{E}}\right)^{2}: \Lambda^{*} \otimes \mathcal{E} \longrightarrow \Lambda^{*+2} \otimes \mathcal{E}
$$

vérifie

$$
\forall \omega \in \Lambda^{*}, \forall s \in C^{\infty}(\mathcal{E}), \quad\left(\nabla^{\mathcal{E}}\right)^{2}(\omega \otimes s)=\omega \otimes\left(\nabla^{\mathcal{E}}\right)^{2}(s) .
$$

Par conséquent $\left(\nabla^{\mathcal{E}}\right)^{2}$ et $\mathrm{d} a$ commutent.

BULLETIN DE LA SOCIÉTÉ MATHÉMATIQUe DE FRANCE 
On vient donc de prouver que $\sigma_{0}\left((\mathrm{~d} a)^{(1)}\right)=0$. Par une récurrence évidente, on obtient $\sigma_{0}(\mathrm{~d} a)^{(k)}=0$ pour tout $k \in \mathbb{N}$. Comme $Q$ contient au moins un tel facteur, on peut conclure que $\sigma_{0}(Q)=0$.

Si $k=0$ alors $\sigma_{0}([D, a])=\mathrm{d} a$ et la formule pour le produit donne l'expression du lemme.

Fin de la preuve de la proposition 5.2. - Notons $Q_{u}$ l'opérateur obtenu en remplaçant $D$ par $u D(u>0)$. On vérifie immédiatement que $Q_{u}=u^{n+2|k|} Q$. Même notation pour $A=\mathrm{e}^{-\widetilde{D}^{2}}$, on pose

$$
A_{u}:=\mathrm{e}^{-u^{2} \widetilde{D}^{2}} .
$$

Le calcul qui suit est maintenant entièrement justifié :

$$
\begin{aligned}
& \operatorname{Str} Q \mathrm{e}^{-t \mathcal{D}^{2}}=\operatorname{Str} Q \mathrm{e}^{-t \widetilde{D}^{2}} \psi \\
& =\int_{T^{*} X} \sigma_{t^{-\frac{1}{2}}}\left(Q \mathrm{e}^{-t \widetilde{D}^{2}} \psi\right)(x, \xi) \mathrm{d} x \mathrm{~d} \xi \\
& =t^{-\left(\frac{1}{2} n+|k|\right)} \int_{T^{*} X} \sigma_{t^{-\frac{1}{2}}}\left(Q_{\sqrt{t}} A_{\sqrt{t}} \psi\right)(x, \xi) \mathrm{d} x \mathrm{~d} \xi \\
& =t^{-\left(\frac{1}{2} n+|k|\right)} \int_{T^{*} X} \sigma_{0}(Q A \psi)(x, \xi) \mathrm{d} x \mathrm{~d} \xi \\
& =t^{-\left(\frac{1}{2} n+|k|\right)} \int_{T^{*} X} \sigma_{0}(Q) * \sigma_{0}(A \psi)(x, \xi) \mathrm{d} x \mathrm{~d} \xi+O\left(t^{-\left(\frac{1}{2} n+|k|\right)+\frac{1}{2}}\right) \\
& =\left\{\begin{array}{l}
O\left(t^{-\left(\frac{1}{2} n+|k|\right)+\frac{1}{2}}\right) \quad \text { si }|k|>0, \\
t^{-\frac{1}{2} n} \nu_{n} \int_{X} a_{0} \mathrm{~d} a_{1} \wedge \cdots \wedge \mathrm{d} a_{n} \hat{A}(X) \wedge \mathrm{Ch}(E)+O\left(t^{\frac{1}{2}(-n+1)}\right), \text { si } k=0 .
\end{array}\right.
\end{aligned}
$$

Dans la cinquième ligne, on a utilisé (voir [13])

$$
\sigma_{t^{-\frac{1}{2}}}\left(Q_{\sqrt{t}} A_{\sqrt{t}} \psi\right)(x, \xi)=\sigma_{0}(Q A \psi)(x, \xi)+O\left(t^{\frac{1}{2}}\right),
$$

où $O\left(t^{\frac{1}{2}}\right)$ est d'ordre $-\infty$ et nécessairement à support compact en $x$, donc on peut effectuer son intégrale sur $T^{*} X$ et récupérer un $O\left(t^{\frac{1}{2}}\right)$.

Il reste à préciser ce qui se passe pour $n=0$ dans le théorème 5.1. La composante $\varphi_{0}(a+\lambda)$ est donnée par le terme constant du développement asymptotique en $t=0$ de

$$
\operatorname{Str}\left((a+\lambda) \mathrm{e}^{-t \mathcal{D}^{2}}\right)=\operatorname{Str}\left(a \mathrm{e}^{-t \mathcal{D}^{2}}\right)+\lambda \operatorname{Str} \mathrm{e}^{-t \mathcal{D}^{2}} .
$$

Les opérateurs $\mathcal{D}$ étant $\mathbb{Z}_{2}$-gradués, on a la formule de Mc Kean-Singer :

$$
\forall t>0, \quad \operatorname{Str} \mathrm{e}^{-t \mathcal{D}^{2}}=\operatorname{Ind}\left(\mathcal{D}_{+}\right) .
$$

TOME $129-2001-\mathrm{N}^{\mathrm{O}} 4$ 
D'autre part, comme la fonction $a$ est $C^{\infty}$ à support compact, la proposition 5.2 s'applique et on obtient

$$
\operatorname{Str}\left(a \mathrm{e}^{-t \mathcal{D}^{2}}\right)=\int_{X} a \hat{A}(X) \wedge \operatorname{Ch}(E) .
$$

Finalement :

$$
\varphi_{0}(a+\lambda)=\varphi_{0}(a)+\varphi(\lambda)=\int_{X} a \hat{A}(X) \wedge \operatorname{Ch}(E)+\lambda \operatorname{Ind}\left(D_{+}\right)_{\min } .
$$

Cela termine également la preuve du théorème 5.1.

5.3. Générateurs de la $K$-homologie. - Le théorème 5.1 donne un résultat de généricité. Notons $\mathcal{E}_{D}(X)$ la collection des triplets spectraux sur $\mathcal{A}$ obtenus à partir des extensions auto-adjointes $\mathcal{D}^{\prime}$ et $\mathcal{D}^{\prime \prime}$ des opérateurs de Dirac $D_{E}$ à coefficients dans des fibrés.

Proposition 5.5. $-\mathcal{E}_{D}(X)$ engendre $K_{0}\left(X^{c}\right) \otimes \mathbb{C}$.

Démonstration. - Rappelons les deux isomorphismes de Chern que nous allons utiliser :

$$
\begin{aligned}
K^{0}(\bar{X}) & \otimes \mathbb{C} \longrightarrow H^{\mathrm{ev}}(\bar{X} ; \mathbb{C}), \\
K_{0}\left(X^{c}\right) & \otimes \mathbb{C} \longrightarrow H_{\mathrm{ev}}\left(X^{c} ; \mathbb{C}\right) .
\end{aligned}
$$

La forme différentielle $\hat{A}(X)$ étant inversible dans l'anneau des formes différentielles $C^{\infty}$ sur $X$, la dualité de Poincaré pour les variétés à bord entraîne que l'application :

$$
\begin{aligned}
H^{\mathrm{ev}}(\bar{X} ; \mathbb{C}) & \longrightarrow \bar{H}_{\mathrm{ev}}\left(X^{c} ; \mathbb{C}\right) \\
\omega & \longmapsto \int_{X} \hat{A}(X) \wedge \omega \wedge .
\end{aligned}
$$

qui à $\omega$ associe le courant $C^{\infty}$ représenté par $\hat{A}(X) \wedge \omega$ (voir [26]), est un isomorphisme $\left(\bar{H}_{\mathrm{ev}}\right.$ désigne l'homologie réduite de $X^{c}$ et coïncide avec l'homologie à supports fermés de $X$ ).

Comme, d'une part, les fibrés $E$ utilisés dans la définition de $\mathcal{E}_{D}(X)$ engendrent le $K^{0}$ de la variété à bord $\bar{X}$ et d'autre part l'isomorphisme (36) est valable pour les versions réduites de $K_{0}$ et $H_{\mathrm{ev}}$, on obtient déjà que la restriction des éléments de $\mathcal{E}_{D}(X)$, qui sont des $K$-cycles sur $\mathcal{A}$, à $\bar{A}=\mathcal{A} / \mathbb{C}$ engendrent $\bar{K}_{0}\left(X^{c}\right) \otimes \mathbb{C}$. Pour vérifier qu'ils engendrent bien $K_{0}\left(X^{c}\right) \otimes \mathbb{C}$, il suffit de trouver $\mathcal{D}_{E}$ appartenant à $\mathcal{E}_{D}(X)$ tel que $\operatorname{Ind}\left(\mathcal{D}_{E_{+}}\right) \neq 0$.

Considérons simplement l'opérateur de Dirac $D$ sur $X$. Les extensions fermées de $D_{+}$sont connues par le travail de J. Brüning et R. Seeley [6] et

$$
\operatorname{Ind}\left(D_{+}\right)_{\max }-\operatorname{Ind}\left(D_{+}\right)_{\min }=\sum_{\lambda \in]-\frac{1}{2}, \frac{1}{2}[} n_{\lambda},
$$

BULletin DE LA SOCiÉtÉ MATHÉMATIQUE DE FRANCE 
où $n_{\lambda}$ est la multiplicité de $\lambda$ comme valeur propre de $D_{N}$, l'opérateur de Dirac induit sur $N$. Or, si l'on multiplie la métrique de $N$ par une constante positive $c^{2}$, les valeurs propres de $D_{N}$ sont multipliées par $c^{-1}$. On peut donc supposer, quitte à dilater suffisamment la métrique de $N$, que Spec $\left.D_{N} \cap\right]-\frac{1}{2}, \frac{1}{2}[\neq \varnothing$, donc qu'un des deux indices dans (37) est non nul. Alors, soit $\mathcal{D}^{\prime}$ soit $\mathcal{D}^{\prime \prime}$ répond au problème.

\section{BIBLIOGRAPHIE}

[1] Baum (P.) \& Douglas (R.) - K-homology and index theory, Proc. Sympos. Pure Math., t. 38 (1982), no. 1, pp. 117-173.

[2] Berline (N.), Getzler (E.) \& Vergne (M.) - Heat kernels and Dirac operators, Comprehensive Studies in Math., vol. 298, Springer-Verlag, 1991.

[3] Bost (J.B.) - Principe d'Oka, K-théorie et systèmes dynamiques non commutatifs, Invent. Math., t. 101 (1990), pp. 261-333.

[4] Brüning (J.) \& Lesch (M.) - On the spectral geometry of algebraic curves, J. reine angew Math., t. 474 (1996), pp. 25-66.

[5] Brüning (J.) \& Seeley (R.) - Regular singular asymptotics, Advances in Math., t. 58 (1985), pp. 133-148.

[6] _ An index theorem for first order regular singular operators, Amer. J. Math., t. 110 (1988), pp. 659-714.

[7] Chan (S.W.) - Periodic cyclic cohomology Chern character for pseudomanifolds with one singular stratum, Proc. AMS, t. 126 (1998), no. 3, pp. 669-675.

[8] Cheeger (J.) - On the spectral geometry of spaces with cone-like singularities, Proc. Nat. Acad. Sci. USA, t. 76 (1979), pp. 2103-2106.

[9] _ Spectral geometry of singular riemannian spaces, J. Diff. Geom., t. 18 (1983), pp. 575-657.

[10] Chou (A.W.) - The Dirac operator on spaces with conical singularities and positive scalar curvatures, Trans. AMS, t. 289 (1985), no. 1, pp. 1-40.

[11] Connes (A.) - Noncommutative differential geometry, Pub. IHES, t. 62 (1985), pp. 257-360.

[12] _ Noncommutative Geometry, Academic Press, 1994.

[13] Connes (A.) \& Moscovici (H.) - Cyclic cohomology, Novikov conjecture and hyperbolic groups, Topology, t. 29 (1990), pp. 345-388.

[14] _ The local index formula in noncommutative geometry, GAFA, t. 5 (1995), no. 2, pp. 174-243.

[15] Cuntz (J.) \& Quillen (R.) - On excision in periodic cyclic cohomology II : the general case, C.R. Acad Sc. Paris Série I, t. 318 (1994), pp. 11-12.

TOME $129-2001-\mathrm{N}^{\mathrm{O}} 4$ 
[16] Getzler (E.) - Pseudodifferential operators on supermanifolds and the Atiyah-Singer index theorem, Commun. Math. Phys., t. 92 (1983), pp. 163178.

[17] Grubb (G.) \& Seeley (R.) - Weakly parametric pseudodifferential operators and Atiyah-Patodi-Singer boundary problems, Invent. Math., t. 121 (1995), no. 3, pp. 481-529.

[18] Jaffe (A.), Lesniewski (A.) \& Osterwalder (K.) - Quantum Ktheory I. The Chern character, Commun. Math. Phy., t. 118 (1988), pp. 114.

[19] Kasparov (G.G.) - Equivariant KK-theory and the Novikov conjecture, Invent. Math., t. 91 (1988), pp. 147-201.

[20] Lawson (H.) \& Michelsohn (M.L.) - Spin geometry, Princeton Math. Series, vol. 38, Princeton University Press, Princeton, New Jersey, 1989.

[21] Lesch (M.) - Deficiency indices for symmetric Dirac operators on manifolds with conic singularities, Topology, t. 32 (1993), no. 3, pp. 611-623.

[22] _ Operators of Fuchs type, conical singularities, and asymptotic methods, B. G. Teubner Verlagsgesellschaft mbH, Stuttgart, 1997.

[23] Lescure (J.-M.) - Triplets spectraux pour les pseudovariétés à singularité conique isolée, Thèse de Doctorat, Université P. Sabatier, 1998.

[24] Melrose (R.B.) - The Atiyah-Patodi-Singer index theorem, Research Notes in Math., vol. 4, A. K. Peters, Massachusetts, 1993.

[25] Moscovici (H.) \& WU (F.) - Straight Chern character for Witt spaces, preprint IHES, 1996.

[26] De Rham (G.) - Variétés différentiables, Publ. math. Univ. Nancago, vol. Actualités scient. indus., 1222, Hermann, Paris, trois. ed. 1973.

[27] Schulze (B. W.) - Boundary value problems and singular pseudodifferential operators, Wiley-Intersciences, 1998.

[28] Shubin (M.A.) - Pseudodifferential operators and spectral theory, Springer Verlag, 1980.

[29] Skandalis (G.) - Kasparov's bivariant K-theory and applications, Expositiones Math., t. 9 (1991), pp. 193-250. 